\title{
A Technique Based on Adaptive Extended Jacobians for Improving the Robustness of the Inverse Numerical Kinematics of Redundant Robots.
}

\author{
Henrique Simas ${ }^{(\circ)}$ \\ Raul Guenther Laboratory of Applied Robotics, Department of Mechanical Engineering \\ Federal University of Santa Catarina, Florianópolis, SC - Brazil - 88040-900 \\ T: +55 48 3721-4020, F: +55 489 9960-5496 \\ henrique.simas@ufsc.br \\ Raffaele Di Gregorio \\ Department of Engineering, \\ University of Ferrara \\ Via Saragat, 1; 44100 Ferrara; Italy \\ T: +39-0532-974828, F: +39-0532-974870 \\ rdigregorio@ing.unife.it
}

$\left({ }^{\circ}\right)$ Corresponding autor

\begin{abstract}
The extended Jacobian is a technique for solving the redundancy of redundant robots. It is based on the definition of secondary tasks, through constraint functions that are added to the mapping between joint rates and end-effector's twist. Several approaches showed its potential, its applications and limitations. In general, the constraint functions are a linear combination of basic functions with constant coefficients. This paper proposes the use of adaptive coefficients in such functions by using the conditioning index of the extended Jacobian as a quality measure. A good conditioning index of the extended Jacobian keeps the robot far from singularities and contributes to the solution of the inverse kinematics. In this paper, initially the extended Jacobian and the proposed algorithm are discussed, then two tests in different circumstances are presented in order to validate the proposal.
\end{abstract}

Keywords: adaptive extended Jacobian, redundant robots, conditioning index, numerical inverse kinematics.

\section{INTRODUCTION}

Redundant robots have an additional number of joints (mobility) that allows us to include additional tasks during its operation. Such class of robots has greater versatility and possibilities of movements in the execution of a task when compared with conventional robots.

The characterization of a robot as redundant is associated with its degree of redundancy $r$ or the difference between the number $n$ of active joints of the robot, which coincides with its degrees of freedom 
(DOF), and the number $m$ of degrees of freedom required to describe a given task, expressed by: $r=n-m[1]$.

The redundancy needs new methods for the solution of forward and inverse kinematics, since classical methods have difficulties and limits for their application. The main methods are based on the use of null space of the Jacobian matrix, such as Pseudoinverse or optimization [1][9][21][33][41][47] and taskpriority [5][8[10][27], based on Euclidean norms, bringing with it the problem of dimensional scales, among others [1][3][6][10]. Also, strategies based on the dynamics [19][33], extension of the Jacobian [1][2][6-8][15][16][34] and evolutionary algorithms [15][37][38] are used, but need the knowledge with certain precision of the dynamic model of the robot. In order to overcome the use of Euclidean norms, the strategy of the extended (or augmented) Jacobian become as proposal based on the definition of secondary tasks in the form of kinematic constraints, or equations, added as supplementary lines to the Jacobian, making it square and allowing its inversion. In general, the extended Jacobian or augmented Jacobian [21] is simple to be implemented and useful, when compared with the method based on differential models. Among the possible secondary tasks, the collision avoidance stands out [6][14][41]. Other tasks aim at solving singularities problems [8][9][11][14][41][50][52] or proposing strategies using optimized CI to keep the redundant robot far from singularities [34][40][42][44][45][49].

The technique of extended Jacobian, is presented as the simplest, because it is based on the addition of lines to the Jacobian matrix, until it becomes square and can be inverted. In general, the number of additional lines is defined according to the degree of redundancy $r$ of the redundant robot. The extended Jacobian has application in both industrial robots [6][22] and mobile robots [13][28]. The additional lines of the extended Jacobian represent kinematic restrictions that involve joints positions or velocities and/or end-effector's coordinates.

The extended Jacobian has a well-known limitation called algorithmic singularities [1][8][27][29], which correspond to the singular postures assumed by the robot that do not belong to its kinematic chain. Some propositions, based on reciprocity [16][20] and task-priority [27][29] overcome the problem of algorithm singularities. Although simple, the use of extended Jacobian requires the continuous monitoring of singularities on tracking trajectories. For such monitoring, the Conditioning Index (CI) [4] allows us to evaluate the distances of the singularities, since it is required the inversion of the extended Jacobian to 
solve the inverse kinematics. CI is also a reference for solutions of inverse kinematics for redundant robots and hyper-redundant robots [41][47][50], dealing with path planning and workspace design [36][40][41][47-49][52], design and dexterity [48][51], joint limits [44], singularities [39][42][48][51] and collision avoidance [40][50], in general leading with optimized solutions [43][45][46], for a desired tasks.

In general the additional lines of the extended Jacobian are composed by basic functions with fixed coefficients. In this paper, we show that by varying the coefficients of such lines, it is possible to increase the CI of the extended Jacobian matrix and, consequently, improve the results obtained in the Jacobian inversion in terms of distance from singularities. The direct consequences of this proposal lie in the fact that increasing the CI of the extended Jacobian for each point of a trajectory increases the Global Conditioning Index (GCI) [4] of the task, as well as minimizes computational efforts in the solution of the inverse kinematics, usually solved by means of numerical algorithms.

This paper proposes a simple and versatile search algorithm that seeks the best task-dependent coefficients that maximize the CI of extended Jacobians applied to redundant robots.

The paper is organized as follows: Section 2 describes the extended Jacobian and its general formulation; Section 3 presents the proposed algorithm; Section 4 presents a case study on a redundant planar robot, and finally Section 5 draws the conclusions.

\section{EXTENDED JACOBIAN}

The extended Jacobian technique emerges as an alternative to the problem of dimensional inconsistency presented in the use of the Moore-Penrose Pseudoinverse [1][3][10].

The extended Jacobian, also known as the task extension method or augmented Jacobian [21], was proposed by Baillieul [2][17] in the 80's with the growth of the computation. The extended Jacobian method is based on the imposition of constraint functions (additional lines) in the form $h_{i}(\mathbf{q})=e_{i}$ contained in the set $\mathbf{h}(\mathbf{q})$, as follows

$$
\mathbf{h}(\mathbf{q})=\left\{\mathrm{h}_{1}(\mathbf{q})=e_{1}, \mathrm{~h}_{2}(\mathbf{q})=e_{2}, \cdots, \mathrm{h}_{r}(\mathbf{q})=e_{r}\right\}
$$


where, $\mathbf{q}=\left(q_{1}, q_{2}, \cdots q_{\mathrm{n}}\right)^{\mathrm{T}}$ is the vector collecting the actuated-joint variables with dimension $\mathrm{n}$ (robot DOF) and $\mathbf{e}=\left(e_{1}, e_{2}, \cdots, e_{\mathrm{r}}\right)^{\mathrm{T}}$ is, in general, a vector of constant values. Such constraint function translates into secondary tasks to be accomplished simultaneously with the effector's displacement.

Thus, given the set of constraint function $\mathbf{h}(\mathbf{q})=\mathbf{e}$, the extended kinematics model is obtained by the combination of the following relationships.

$$
\left\{\begin{array}{c}
\mathrm{f}_{\mathrm{x}}(\mathbf{x})=\mathrm{f}_{\mathrm{q}}(\mathbf{q}) \\
e_{1}=\mathrm{h}_{1}(\mathbf{q}) \\
\quad \vdots \\
e_{\mathrm{r}}=\mathrm{h}_{r}(\mathbf{q})
\end{array}\right.
$$

where the first vector equation is the system of closure equations of the mechanism, which relates the actuated-joint variables (q) and the coordinates of the end-effector in the operating space (x) with dimension $\mathrm{m}$.

The forward or inverse kinematics in Eq. (2) can be solved analytically at any point for simple cases. For complex cases, when $h_{i}(\mathbf{q}), f_{x}(\mathbf{x})$ and $f_{q}(\mathbf{q})$ are differentiable and $\mathbf{e}$ is a constant vector, the forward or inverse kinematics can be solved along a trajectory by numerical integration (in general) for a known initial posture, solving the differential kinematics relationship:

or

$$
\begin{gathered}
{\left[\begin{array}{c}
\mathrm{J}_{\mathrm{x}}(\mathbf{x})_{m \times m} \\
\mathbf{0}_{r \times m}
\end{array}\right]_{n \times m} \dot{\mathbf{x}}=\left[\begin{array}{l}
\mathrm{J}_{\mathrm{q}}(\mathbf{q})_{m \times n} \\
\left.\frac{\partial \mathbf{h}(\mathbf{q})}{\partial \mathbf{q}}\right|_{r \times n}
\end{array}\right]_{n \times n} \dot{\mathbf{q}}} \\
\left\{\mathrm{J}_{\text {ex }}(\mathbf{x})\right\}_{n \times m} \dot{\mathbf{x}}=\left\{\mathrm{J}_{\mathrm{eq}}(\mathbf{q})\right\}_{n \times n} \dot{\mathbf{q}}
\end{gathered}
$$

where $\mathrm{J}_{\mathbf{x}}(\mathbf{x})==\mathrm{df}_{\mathbf{x}}(\mathbf{x}) / \mathrm{d} \mathbf{x}$ and $\mathrm{J}_{\mathrm{q}}(\mathbf{q})=\mathrm{df}_{\mathrm{q}}(\mathbf{q}) / \mathrm{d} \mathbf{q}$ are the Jacobians obtained from the derivative of the expression $\mathrm{f}_{\mathrm{x}}(\mathbf{x})$ and $\mathrm{f}_{\mathrm{q}}(\mathbf{q})$ respectively; $\mathrm{J}_{\mathrm{ex}}(\mathbf{x})$ and $\mathrm{J}_{\mathrm{eq}}(\mathbf{q})$ are the extended Jacobians for the resultant instantaneous forward and inverse kinematics respectively and

$$
\frac{\partial \mathbf{h}(\mathbf{q})}{\partial \mathbf{q}}=\left[\begin{array}{ccc}
\partial \mathrm{h}_{1}(\mathbf{q}) / \partial \mathrm{q}_{1} & \cdots & \partial \mathrm{h}_{1}(\mathbf{q}) / \partial \mathrm{q}_{\mathrm{n}} \\
\vdots & & \vdots \\
\partial \mathrm{h}_{\mathrm{r}}(\mathbf{q}) / \partial \mathrm{q}_{1} & \cdots & \partial \mathrm{h}_{\mathrm{r}}(\mathbf{q}) / \partial \mathrm{q}_{\mathrm{n}}
\end{array}\right]_{\mathrm{r} \times n}
$$

are the additional lines that define the extended Jacobian $\mathrm{J}_{\mathrm{eq}}(\mathbf{q})$, obtained by differentiating each constraint function $h_{i}(\mathbf{q})$ contained in $\mathbf{h}(\mathbf{q})$. 
The condition for the existence of a single solution $\mathbf{q}$ in Eqn. (3) is that $\mathrm{J}_{\mathrm{eq}}(\mathbf{q})$ be non-singular along the trajectory.

The extended Jacobian has the drawback of introducing algorithmic singularities. The algorithmic singularities are related only to the additional lines of $\mathrm{J}_{\mathrm{eq}}(\mathbf{q})$, and not to the own robot's kinematics [1][10][26]. In this sense, the advantages of redundant robots can be limited by false singularities, possibly generated by an inconvenient selection of the constraint functions $\mathbf{h}(\mathbf{q})$ [3][5]. Another limitation presented in the application of extended Jacobian is lack of cyclicity, or repeatability in the solution in the forward or inverse kinematics [1]. Such problem arises when a cyclic trajectory imposed to the end-effector does not correspond to a cyclic trajectory in the joint space. This phenomenon implies that the kinematic behavior of the redundant robots become unpredictable throughout the task. As a way to overcome this problem, the most used set $\mathbf{h}(\mathbf{q})$ is based on the optimization of the total energy [1][2][5][9][16][25][27] in a displacement.

A general form of the constraint function $\mathrm{h}_{\mathrm{i}}(\mathbf{q})$ can be written as follows

$$
\mathrm{h}_{\mathrm{i}}(\mathbf{q})=\sum_{\mathrm{j}=1}^{n} f_{\mathrm{j}} \mathrm{g}_{\mathrm{j}}(\mathbf{q})=e_{\mathrm{i}}
$$

where the terms $g_{j}(\mathbf{q})$ are the basic functions and $f_{j}$ are the weight coefficients for each $h_{i}(\mathbf{q})$ function.

Therefore, for $r h_{i}(\mathbf{q})$ functions, and $n$ actuated joints, we have $r \times n$ coefficients $\left.f_{\mathrm{j}}\right|_{\mathrm{h}_{\mathrm{i}}}\left(f_{\mathrm{j}}\right.$ for simplicity) to be adjusted. Such adjustment of the coefficients $f_{\mathrm{j}}$ can be an exhaustive task, in general done empirically, even being commonly adjusted for constant values as 1 (for simplicity). But simplified or empirical adjustments do not guarantee the best kinematic performance in the use of extended Jacobian.

Considering the complexity of the differential kinematic model, obtained by means of the extended Jacobian, numerical techniques are used for the solution of the inverse position analysis. The most common method is called Resolved Motion Control (RMC)[11-14][23][24], where the differentials $\dot{\mathbf{x}}$ and $\dot{\mathbf{q}}$ vectors from Eq. (4) are approximated by $1^{\text {st }}$ order discrete relations $\dot{\mathbf{x}} \cong \frac{\mathbf{x}_{\mathrm{i}+1}-\mathbf{x}_{\mathrm{i}}}{\Delta t}$ and $\dot{\mathbf{q}} \cong \frac{\mathbf{q}_{\mathrm{i}+1}-\mathbf{q}_{\mathrm{i}}}{\Delta t}$ respectively, where $\mathbf{x}_{\mathrm{i}}\left(\mathbf{x}_{\mathrm{i}+1}\right)$ and $\mathbf{q}_{\mathrm{i}}\left(\mathbf{q}_{\mathrm{i}+1}\right)$ are the positions coordinate's vector of the end-effector and actuated-joint variables vector at instant time $t_{\mathrm{i}}\left(t_{\mathrm{i}+1}\right)$, and $\Delta t=t_{\mathrm{i}+1}-t_{\mathrm{i}}$. 
In the solution of the inverse kinematics (via RMC, or similar method), the inverse of the extended Jacobian is required, and as "far" as possible such Jacobian stays from singularities, better conditioned will be its inverse, and better performance in terms of convergence and computational efforts are obtained. Incorrect adjustments in the coefficients of the constraint functions lead to undesirable situations, such as ill-conditioned Jacobian and consequently numerical instabilities.

In the next section, an adaptive adjustment method is proposed for computing the coefficients $f_{j}$, in such way that maximize a kinematic performance objective function, in this case, the CI [4] which measures the distance from the singularities.

\section{PROPOSED METHODOLOGY}

\subsection{Proposition}

As discussed in previous sections, possible numerical instabilities and unpredictable displacements can occur in the solution to the inverse kinematics using numerical methods, via differential kinematic modeling based on extended Jacobian.

The constraint functions $h_{\mathrm{i}}(\mathbf{q})=e_{\mathrm{i}}$ have the $f_{\mathrm{j}}$ coefficients that can be adjusted in order to improve the kinematic performance so that the extended Jacobian stays as "far" as possible from geometric and algorithmic singularities. For controlling the distance from singularities the CI can be used.

In the inverse kinematics problem, the parameter CI corresponds to the inverse of the condition number [4] of the extended Jacobian $\mathrm{J}_{\mathrm{eq}}(\mathbf{q})$. Adopting the spectral norm [30][31] to compute the condition number, the CI is equal to the ratio between the smallest and the largest singular values of $\mathrm{J}_{\mathrm{eq}}(\mathbf{q})[4]$. The CI ranges from 0 , at singular configurations, to 1 , at isotropic configurations, which are the best configurations [4].

It is observed that the CI values are function of the posture and link lengths of the robot [4], as well as now, the coefficients $f_{\mathrm{j}}$ of the extended Jacobian.

Considering the possible variations of the coefficients $f_{\mathrm{j}}$ within the extended Jacobian, it is observed that it is possible to maximize the value of $\mathrm{CI}$, that is, for the same robot posture it is possible to increase the distance from the singularities, contributing with the numerical algorithms for inverse kinematics. A 
study using a planar redundant robot, that will be presented and discussed in the next section, was done ${ }^{1}$. Using such redundant robot as example in a fixed posture, it is shown in Fig. 1 that there is a maximum value for CI as a function of the coefficients $f_{\mathrm{j}}$.

In the 4DOF redundant robot that will be discussed in section 4 (see Fig. 3), we have 2 coefficients to be adjusted: $\tilde{f}_{4}$ and $\tilde{f}_{6}$ (see Eqs (28) and (29)).. In the evaluation presented in Fig.1, we defined the values of $\tilde{f}_{4}$ and $\tilde{f}_{6}$ ranging from -4 to 4 . In Fig. 1 , it can be seen that the CI can reach the value 0 when $\tilde{f}_{6} \cong 4 / 3 \tilde{f}_{4}$, that is, a poor choice for these coefficients which bring the extended Jacobian near to its singularities, or even take it to a singular condition, independently of the joint positions or robot posture. Also, a symmetry is observed along the line $\tilde{f}_{6}-4 / 3 \tilde{f}_{4} \cong 0$, indicating that, for this example, there are two sets of values for $f_{4}$ and $f_{6}$ that can be chosen.

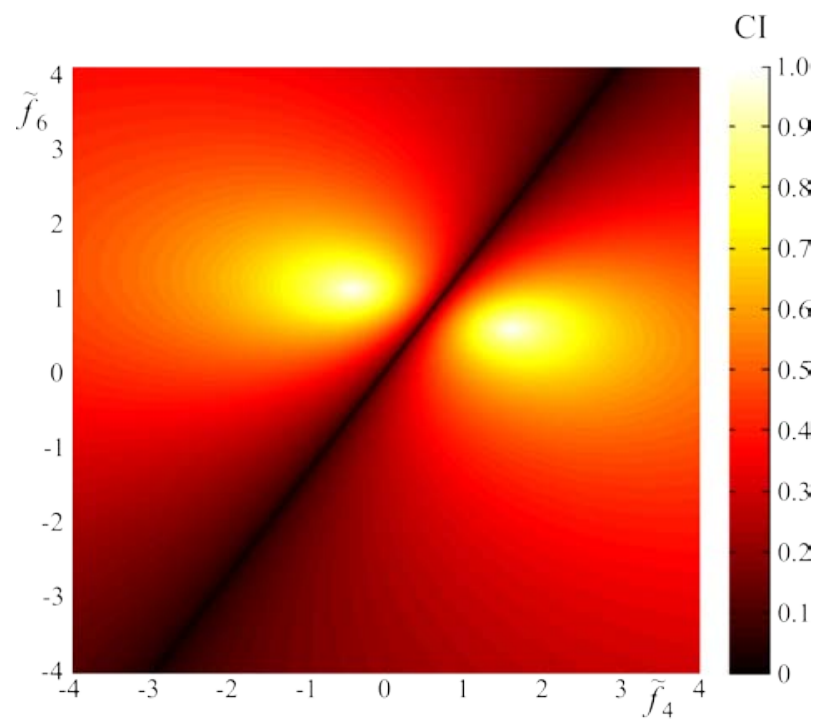

FIGURE 1. GEOMETRIC BEHAVIOR OF THE CONDITIONING INDEX AS A FUNCTION OF THE PARAMETERS $\tilde{f}_{4}$ AND $\tilde{f}_{6}$.

\subsection{Algorithms}

The possibility of increasing the CI of the extended Jacobian and consequently the GCI of the task by the variation of the coefficients $\mathrm{f}_{\mathrm{j}}$, from the constraint functions $\mathbf{h}(\mathbf{q})$ is considered. Here, a simple and versatile search algorithm to do this is proposed and depicted as a flowchart in the Fig.2.

\footnotetext{
${ }^{1}$ In this analysis the following displacements were used: $d_{1}=-0.74$ l.u., $d_{3}=2$ l.u., $d_{4}=1$ l.u. and $d_{6}=2$ l.u. See section 4 and Fig. 3 for more details.
} 


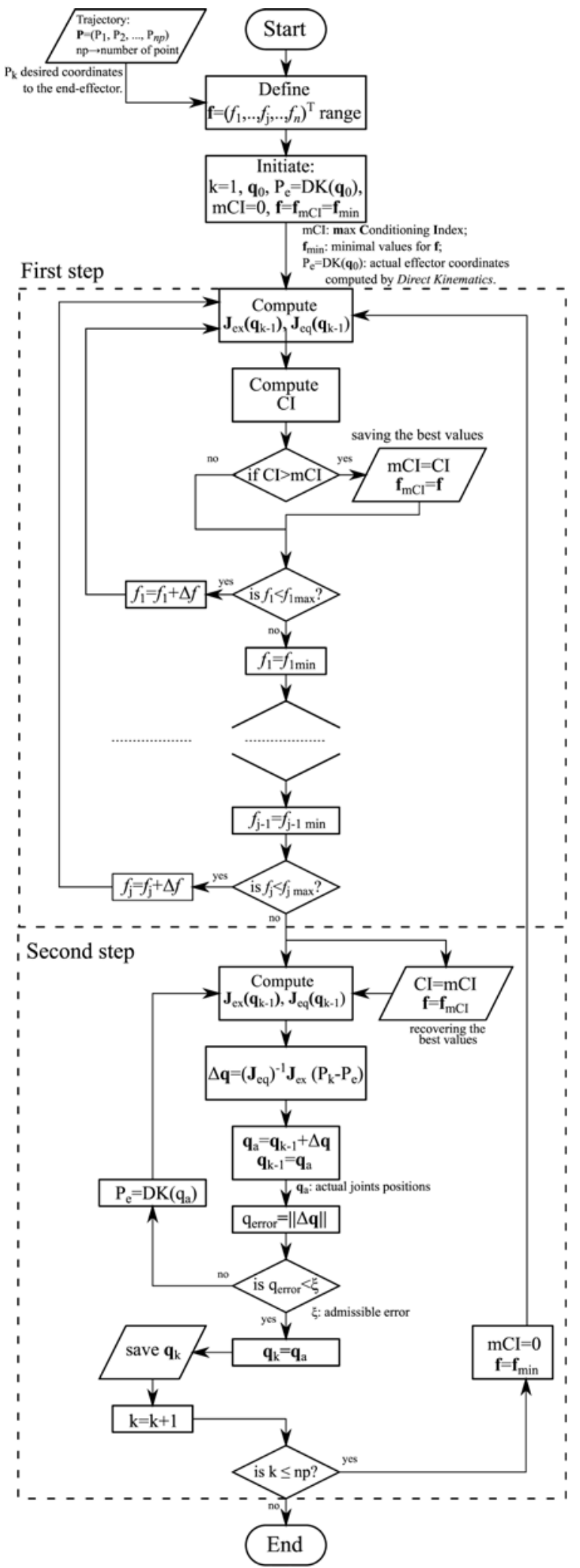

FIGURE 2. FLOWCHART OF THE PROPOSED ALGORITHM. 
Let us consider a desired trajectory defined by a set of coordinates $\mathbf{P}_{\mathrm{k}}$ for $k=1, \ldots, n p$ (where $n p$ is the number of points belonging to the trajectory) to the end-effector and assign the ranges of the $f_{\mathrm{j}}$ values for each function from $\mathbf{h}(\mathbf{q})$. Considering known the initial posture of the robot, the proposed method is based on two-steps algorithm: the first step consists in varying the values of the $f_{\mathrm{j}}$ within its ranges, as function of a desired increment $\Delta$ f, trying to find the values of $\mathbf{f}_{\mathrm{mCI}}$ that yield the maximum (best) value for the CI by the of the $\mathrm{J}_{\mathrm{eq}}(\mathbf{q})$ matrix in the actual robot posture, comparing with the actual maximum $\mathrm{CI}$ (mCI). At the end the found values of $\mathbf{f}=\mathbf{f}_{\mathrm{mCI}}$ and $\mathrm{CI}$ are stored.

Considering that the values of $\mathbf{f}$ ensure the best $\mathrm{CI}$ for $\mathrm{J}_{\mathrm{eq}}(\mathbf{q})$ matrix (i.e., the maximum distance from singularities) and knowing the actual end-effector pose $\left(\mathbf{P}_{\mathrm{e}}\right)$ and the next end-effector pose in the trajectory (next $\mathbf{P}_{\mathrm{k}}$ ), the second step consists in computing the actuated-joint variables of the robot by means of the numerical integration (RMC) from Eq.(4), that is, by isolating $\dot{\mathbf{q}}$, the first order approximations for $\dot{\mathbf{q}} \cong \Delta \mathbf{q} / \Delta t$ and $\dot{\mathbf{x}} \cong\left(\mathbf{P}_{\mathrm{k}}-\mathbf{P}_{\mathrm{e}}\right) / \Delta t$ are used in Eq. (4) and the next $\mathbf{q}$ is determined. Defining a desired and admissible approximation error $\xi$ to the norm of the active joint positions vector (stopping criterion), when the procedures for computing the new posture of the robot are completed, the process restarts from the first step to the current robot posture.

At the end of the process, the GCI [4][31] is computed as performance index for the entire executed task. Figure 2 presents a detailed flowchart of the algorithm, indicating the procedures, the flow of variables and which variables are stored for the purpose of simulation and presentation of results. In the flowchart, some comments are added in order to evidence the understanding of the details of some procedures.

\subsection{Stability analysis}

For the convergence analysis, let's consider the differential relation to the extended Jacobian presented in Eq. (4) rewritten as

$$
\dot{\tilde{\mathbf{x}}}=\mathbf{M} \dot{\mathbf{q}}
$$

where $\dot{\tilde{\mathbf{x}}}=\left\{\mathrm{J}_{\mathrm{ex}}(\mathbf{x})\right\}_{\mathrm{n} \times \mathrm{m}} \dot{\mathbf{x}}$ and $\mathbf{M}=\left\{\mathrm{J}_{\mathrm{eq}}(\mathbf{q})\right\}_{\mathrm{n} \times \mathrm{n}}$.

The inverse matrix relation is given by: 


$$
\dot{\mathbf{q}}=\mathbf{M}^{-1} \dot{\tilde{\mathbf{x}}}
$$

Using the norms inequalities relations [30] for Eqs. (7) and (8) we have that:

$$
\begin{gathered}
\|\dot{\tilde{\mathbf{x}}}\| \leq\|\mathbf{M}\|\|\dot{\mathbf{q}}\| \\
\|\dot{\mathbf{q}}\| \leq\left\|\mathbf{M}^{-1}\right\|\|\dot{\tilde{\mathbf{x}}}\|
\end{gathered}
$$

$\dot{\tilde{\mathbf{x}}}$ and $\dot{\mathbf{q}}$ vectors from Equations (7) and (8) are approximated by the $1^{\text {st }}$ order discrete relations $\dot{\tilde{\mathbf{x}}} \cong \frac{\tilde{\mathbf{x}}_{\mathrm{i}+1}-\tilde{\mathbf{x}}_{\mathrm{i}}}{\Delta t}=\frac{\Delta \tilde{\mathbf{x}}_{\mathrm{i}}}{\Delta t}$ and $\dot{\mathbf{q}} \cong \frac{\mathbf{q}_{\mathrm{i}+1}-\mathbf{q}_{\mathrm{i}}}{\Delta t}=\frac{\Delta \mathbf{q}_{\mathrm{i}}}{\Delta t}$ respectively, where $\tilde{\mathbf{x}}_{\mathrm{i}}\left(\tilde{\mathbf{x}}_{\mathrm{i}+1}\right)$ and $\mathbf{q}_{\mathrm{i}}\left(\mathbf{q}_{\mathrm{i}+1}\right)$ are the positions coordinate's vector of the end-effector and the actuated-joint variables vector at instant time $t_{i}\left(t_{i+1}\right)$, and $\Delta t=t_{\mathrm{i}+1}-t_{\mathrm{i}}$. Thus:

or

$$
\begin{gathered}
\|\Delta \tilde{\mathbf{x}}\| \leq\|\mathbf{M}\|\|\Delta \mathbf{q}\| \\
\frac{\|\Delta \mathbf{q}\|}{\|\Delta t\|} \leq\left\|\mathbf{M}^{-1}\right\| \frac{\|\Delta \tilde{\mathbf{x}}\|}{\|\Delta t\|} \text { or }\|\Delta \mathbf{q}\| \leq\left\|\mathbf{M}^{-1}\right\|\|\Delta \tilde{\mathbf{x}}\|
\end{gathered}
$$

From Eq. (11) the following relations are valid [30]

$$
\tilde{\mathbf{x}}=\mathbf{M q} \quad \text { or }\|\tilde{\mathbf{x}}\| \leq\|\mathbf{M}\|\|\mathbf{q}\| \text { and }\|\mathbf{q}\| \geq \frac{\|\tilde{\mathbf{x}}\|}{\|\mathbf{M}\|}
$$

Dividing both sides of Eq. (12) by $\|\mathbf{q}\|$ and using Eq. (13), we have the following result

$$
\frac{\|\Delta \mathbf{q}\|}{\|\mathbf{q}\|} \leq \frac{\left\|\mathbf{M}^{-1}\right\|\|\Delta \tilde{\mathbf{x}}\|}{\frac{\|\tilde{\mathbf{x}}\|}{\|\mathbf{M}\|}}
$$

thus

$$
\frac{\|\Delta \mathbf{q}\|}{\|\mathbf{q}\|} \leq\left\|\mathbf{M}^{-1}\right\|\|\mathbf{M}\| \frac{\|\Delta \tilde{\mathbf{x}}\|}{\|\tilde{\mathbf{x}}\|}
$$

The value $\left\|\mathbf{M}^{-1}\right\|\|\mathbf{M}\|$ is the condition number of the matrix $\mathbf{M}$ and can be computed by [30]

$$
\left\|\mathbf{M}^{-1}\right\|\|\mathbf{M}\|=\frac{\tau_{\max }}{\tau_{\min }}=\mathrm{k}_{\mathrm{M}}
$$

where $\tau_{\max }$ and $\tau_{\min }$ are the maximum and minimum singular values of $\mathbf{M}$ and $\mathrm{k}_{\mathrm{M}}$ is the condition number. Thus we have 


$$
\frac{\|\Delta \mathbf{q}\|}{\|\mathbf{q}\|} \leq \mathrm{k}_{\mathrm{M}} \frac{\|\Delta \tilde{\mathbf{x}}\|}{\|\tilde{\mathbf{x}}\|}
$$

The CI is obtained by the relation $1 / \mathrm{k}_{\mathrm{M}}$ [30]. Thus for values sufficiently small for $\|\Delta \tilde{\mathbf{x}}\|$, and considering that the proposed algorithm aims to maximize the CI, it implies values for minimized $\mathrm{k}_{\mathrm{M}}$, the norm of $\|\Delta \mathbf{q}\|$ with respect to $\|\mathbf{q}\|$ will always be limited by the resultant values on the right side of the Eq.(17). By this analysis we conclude that the proposed algorithm is stable and converges.

\section{CASE STUDY}

A case study was developed using a redundant planar robot. The objective is to evaluate the performance and limitation of the proposed methodology.

\subsection{DOF Redundant planar robot}

In order to evaluate and validate the proposed algorithm a planar robot is used. The planar robot presented in Fig. 3 is composed by 7 joints and 7 links, which implies that the number of actuated joint variables, or the mobility computed by means of Grübler's mobility criterion [26], is 4 (4DOF). Choosing the four P-pairs as actuated joints $d_{1}, d_{3}, d_{4}$ and $d_{6}$ are the actuated-joint variables; whereas, $\theta_{2}, \theta_{5}$, and $\theta_{7}$ are the joint variables of the three not-actuated (passive) R-pairs . The outputs are the Cartesian coordinates of the point $\mathbf{E}=(x, y)^{\mathrm{T}}$ measure in $\mathrm{O}_{\mathrm{b}}-\mathrm{x}_{\mathrm{b}} \mathrm{y}_{\mathrm{b}}$.

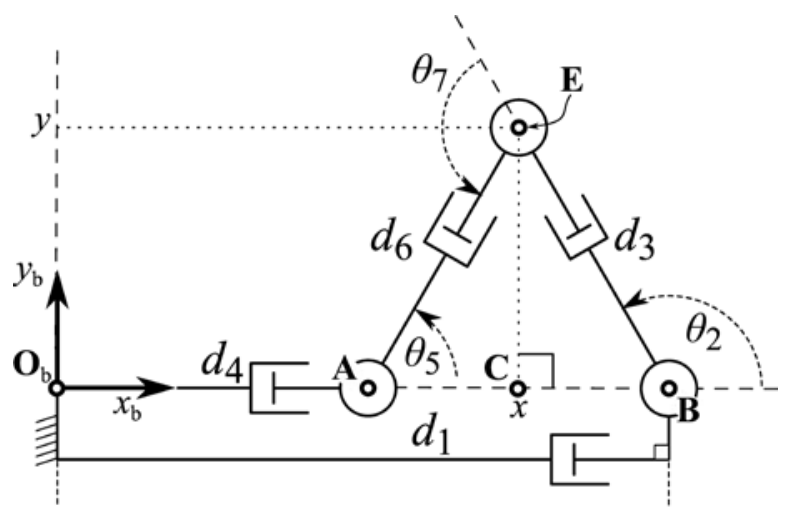

FIGURE 3. PLANAR 4DOF REDUNDANT ROBOT. 
The $4 \mathrm{DOF}$ redundant robot is composed by two legs defined by the points $\mathbf{O}_{\mathrm{b}}, \mathbf{A}, \mathbf{B}, \mathbf{C}$ and $\mathbf{E}$. In the first leg, $d_{1}$ is the actuated-joint variable of the first P-pair and is equal to the length of the segment $\mathbf{B} \mathbf{O}_{\mathrm{b}}$; whereas, $d_{3}$ is the actuated-joint variable of the first P-pair and is equal to the length of the segment $\mathbf{E B}$. In the second $d_{4}$ is the actuated-joint variable of the first P-pair and is equal to the length of the segment $\mathbf{A} \mathbf{O}_{\mathrm{b}}$; whereas, $d_{6}$ is the actuated-joint variable of the second P-pair and is equal to the length of the segment $\mathbf{A B}$. The angles $\theta_{2}, \theta_{5}$, and $\theta_{7}$ (i.e., the passive-joint variables of the three R-pairs) are all counterclockwise, and they are operationally defined in Fig. 3.

$\mathbf{q}=\left(d_{1}, d_{3}, d_{4}, d_{6}\right)^{\mathrm{T}}$ is the vector of the actuated-joint variables, hence, $n=4$; whereas, the coordinates of the point $\mathbf{E}=(x, y)^{\mathrm{T}}$, are the output variables and correspond to the coordinates of a possible end-effector (e.g., the pen of plotter) fixed to the pin of the $\mathrm{R}$ pair centered at E. Thus $m=2$, and the degree of redundancy is $r=n-m=4-2=2$.

With reference to Fig. 3, the right triangles ACE and CBE provide the following relationships:

$$
\begin{aligned}
& d_{3}{ }^{2}=y^{2}+\left(d_{1}-x\right)^{2} \\
& d_{6}{ }^{2}=y^{2}+\left(x-d_{4}\right)^{2}
\end{aligned}
$$

Which, after some simple algebraic manipulation, yield:

$$
\begin{aligned}
& x=\frac{d_{1}^{2}-d_{3}^{2}-d_{4}{ }^{2}+d_{6}{ }^{2}}{2\left(d_{1}-d_{4}\right)} \\
& y^{2}=d_{3}{ }^{2}-\left(\frac{\left(d_{1}-d_{4}\right)^{2}+d_{3}{ }^{2}-d_{6}{ }^{2}}{2\left(d_{1}-d_{4}\right)}\right)^{2}=d_{6}{ }^{2}-\left(x-d_{4}\right)^{2}=d_{3}{ }^{2}-\left(d_{1}-x\right)^{2}
\end{aligned}
$$

Using (20) and (21) a planar workspace was computed by fixing $d_{1}$ and $d_{4}$, and varying $d_{3}$ and $d_{6}$. The resultant workspace is shown in Fig. 4.

By differentiating Eqs. (20) and (21), the following mapping between the infinitesimal displacements of the end-effector and the actuated-joint rates is obtained, in Eq. (22).

$$
\begin{aligned}
\dot{x}\left(d_{1}-d_{4}\right)=\left(d_{1}-x\right) \dot{d}_{1}-d_{3} \dot{d}_{3}-\left(d_{4}-x\right) \dot{d}_{4}+d_{6} \dot{d}_{6} \\
2 y\left(d_{1}-d_{4}\right)^{2} \dot{y}=\left(-2 y^{2}+d_{3}{ }^{2}+d_{6}{ }^{2}-\left(d_{1}-d_{4}\right)^{2}\right)\left(d_{1}-d_{4}\right) \dot{d}_{1}+\left(-d_{3}{ }^{2}+d_{6}{ }^{2}+\left(d_{1}-d_{4}\right)^{2}\right) d_{3} \dot{d}_{3}-\ldots \\
\ldots-\left(-2 y^{2}+d_{3}{ }^{2}+d_{6}{ }^{2}-\left(d_{1}-d_{4}\right)^{2}\right)\left(d_{1}-d_{4}\right) \dot{d}_{4}+\left(d_{3}{ }^{2}-d_{6}{ }^{2}+\left(d_{1}-d_{4}\right)^{2}\right) d_{6} \dot{d}_{6}
\end{aligned}
$$




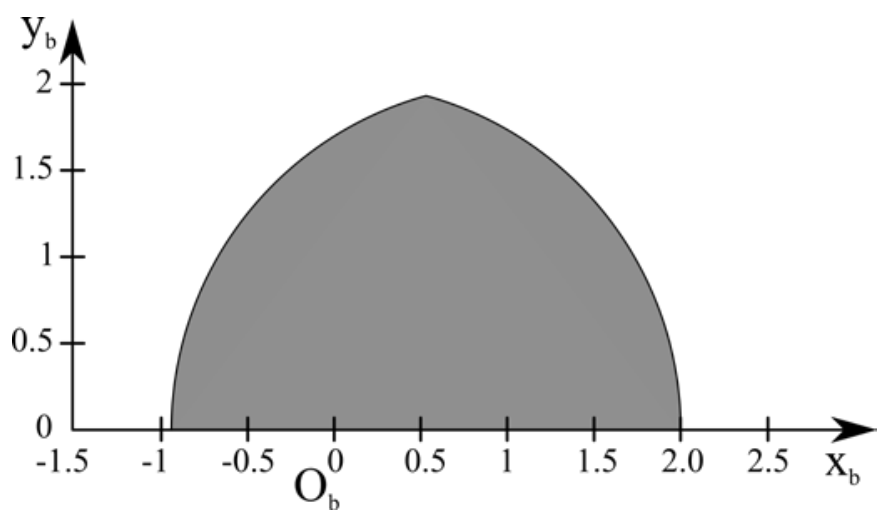

FIGURE 4. 4DOF WORKSPACE FOR $d_{1}=1.065 d_{4}=0$ WITH $0<\left\{d_{3}, d_{5}\right\}<2$.

The matrix form of the Eq.(22) is

$$
\left[\begin{array}{cc}
\left(d_{1}-d_{4}\right) & 0 \\
0 & 2\left(d_{1}-d_{4}\right)^{2} y
\end{array}\right]\left[\begin{array}{c}
\dot{x} \\
\dot{y}
\end{array}\right]=\left[\begin{array}{cccc}
-\left(x-d_{1}\right) & -d_{3} & x-d_{4} & d_{6} \\
k_{\mathrm{a}} & k_{\mathrm{b}} d_{3} & -k_{\mathrm{a}} & k_{\mathrm{c}} d_{6}
\end{array}\right]\left[\begin{array}{c}
\dot{d}_{1} \\
\dot{d}_{3} \\
\dot{d}_{4} \\
\dot{d}_{6}
\end{array}\right]
$$

or

\section{$\mathbf{N} \dot{\mathbf{x}}=\mathbf{M d}$}

where $k_{\mathrm{a}}=\left(-2 y^{2}+d_{3}{ }^{2}+d_{6}{ }^{2}-\left(d_{1}-d_{4}\right)^{2}\right)\left(d_{1}-d_{4}\right), k_{\mathrm{b}}=\left(-d_{3}{ }^{2}+d_{6}{ }^{2}+\left(d_{1}-d_{4}\right)^{2}\right)$

$k_{\mathrm{c}}=\left(d_{3}{ }^{2}-d_{6}{ }^{2}+\left(d_{1}-d_{4}\right)^{2}\right), \dot{\mathbf{x}}=\left[\begin{array}{ll}\dot{x} & \dot{y}\end{array}\right]^{\mathrm{T}}$ and $\dot{\mathbf{d}}=\left[\begin{array}{llll}\dot{d}_{1} & \dot{d}_{3} & \dot{d}_{4} & \dot{d}_{6}\end{array}\right]^{\mathrm{T}}$.

As a redundant robot, the analysis of singularities can be evaluated using the method described in [35]. Therefore, we have type-I [4] singularities when $d_{3}=0$ and $d_{6}=0$, type-II singularities when $d_{1}-d_{4}=$ $d_{3}+d_{6}$ and finally type III singularities when $d_{1}-d_{4}=0$.

Since we have the degree of redundancy $r=2$, the Jacobian became invertible (or square) by the addition of two constraint function, or: $\mathbf{h}(\mathbf{q})=\left\{\mathrm{h}_{1}(\mathbf{q})=e_{1}, \mathrm{~h}_{2}(\mathbf{q})=e_{2}\right\}$.

Based on the minimization of the quadratic cost functional of joint rates [1][21], the following constraint function $\mathbf{h}(\mathbf{q})=\mathbf{e}$ relating the displacements of the P-pairs are selected.

$$
\begin{aligned}
& \mathrm{h}_{1}(\mathbf{d})=f_{1} d_{1}{ }^{2}+f_{4} d_{4}{ }^{2}=e_{1} \\
& \mathrm{~h}_{2}(\mathbf{d})=f_{3} d_{3}{ }^{2}+f_{6} d_{6}{ }^{2}=e_{2}
\end{aligned}
$$

where for each constraint equation was selected $\mathrm{g}_{\mathrm{j}}\left(\mathrm{q}_{\mathrm{j}}\right)=\mathrm{q}_{\mathrm{j}}{ }^{2}$ and $\mathbf{q}=\mathbf{d}=\left[\begin{array}{llll}d_{1} & d_{3} & d_{4} & d_{6}\end{array}\right]^{\mathrm{T}}$.

The time derivatives of Eqs. (24) and (25) give 


$$
\begin{aligned}
& \frac{\mathrm{dh}_{1}(\mathbf{d})}{\mathrm{d} t}=f_{1} d_{1} \dot{d}_{1}+f_{4} d_{4} \dot{d}_{4}=0 \\
& \frac{\mathrm{dh}_{2}(\mathbf{d})}{\mathrm{d} t}=f_{3} d_{3} \dot{d}_{3}+f_{6} d_{6} \dot{d}_{6}=0
\end{aligned}
$$

Then the division of Eqs. (26) and (27) by $f_{1}$ and $f_{3}$, respectively, yields

$$
\begin{aligned}
& d_{1} \dot{d}_{1}+\tilde{f}_{4} d_{4} \dot{d}_{4}=0 \\
& d_{3} \dot{d}_{3}+\tilde{f}_{6} d_{6} \dot{d}_{6}=0
\end{aligned}
$$

where $\tilde{f}_{4}$ and $\tilde{f}_{6}$ represent the relation $f_{4} / f_{1}$ and $f_{6} / f_{3}$ respectively.

Finally, adding the constraint Eqs. (28) and (29) to the differential model (23) the following extended model:

$$
\left[\begin{array}{cc}
\left(d_{1}-d_{4}\right) & 0 \\
0 & 2\left(d_{1}-d_{4}\right)^{2} y \\
0 & 0 \\
0 & 0
\end{array}\right]\left[\begin{array}{c}
\dot{x} \\
\dot{y}
\end{array}\right]=\left[\begin{array}{cccc}
-\left(x-d_{1}\right) & -d_{3} & x-d_{4} & d_{6} \\
k_{\mathrm{a}} & k_{\mathrm{b}} d_{3} & -k_{\mathrm{a}} & k_{\mathrm{c}} d_{6} \\
d_{1} & 0 & \tilde{f}_{4} d_{4} & 0 \\
0 & d_{3} & 0 & \tilde{f}_{6} d_{6}
\end{array}\right]\left[\begin{array}{c}
\dot{d}_{1} \\
\dot{d}_{3} \\
\dot{d}_{4} \\
\dot{d}_{6}
\end{array}\right]
$$

or

\section{$\tilde{\mathbf{N}} \dot{\mathbf{x}}=\tilde{\mathbf{M}} \dot{\mathbf{d}}$}

A simplified differential relationship can be reached by isolating $\dot{d}_{1}$ and $\dot{d}_{3}$ (considering $d_{1} \neq 0$ and $d_{3} \neq 0$ ) in Eqs. (28) and (29) and substituting them in Eq. (30). In so doing, the following reduced matrix form is obtained:

$$
\left[\begin{array}{cc}
d_{1}\left(d_{1}-d_{4}\right) & 0 \\
0 & 2 d_{1}\left(d_{1}-d_{4}\right)^{2} y
\end{array}\right]\left[\begin{array}{l}
\dot{x} \\
\dot{y}
\end{array}\right]=\left[\begin{array}{cc}
-d_{1} d_{4}\left(1+\tilde{f}_{4}\right)+x\left(d_{1}+d_{4} \tilde{f}_{4}\right) & -d_{1} d_{6}\left(1+\tilde{f}_{6}\right) \\
-k_{\mathrm{a}}\left(d_{1}+\tilde{f}_{4} d_{4}\right) & d_{1} d_{6}\left(k_{\mathrm{c}}-k_{\mathrm{b}} \tilde{f}_{6}\right)
\end{array}\right]\left[\begin{array}{l}
\dot{d}_{4} \\
\dot{d}_{6}
\end{array}\right]
$$

\subsection{Validation of the algorithm}

In order to evaluate the performance of the proposed algorithm, two numerical tests were implemented using a laptop with an Intel Core i7 CPU (2.7GHz, 8,00Gb RAM).

Such numerical tests were developed with the purpose of evaluating the behavior of the proposed extended Jacobian, under two different circumstances. The first one extracts a rectangular area of the total workspace area of the redundant robot (Fig. 4) and performs a zigzag task with 1600 points, which sweeps 
40 equidistant lines with 40 equidistant points. The second test make the end effector (i.e., point $\mathbf{E}$ ) track a circular path, for 12 laps which are discretized in a total of 4000 points. The below reported results were obtained by varying the values of the $\tilde{f}_{4}$ and $\tilde{f}_{6}$ coefficients in the interval [-4, 4], in each test.

\subsubsection{Sweeping a rectangular area - Test 1}

For this test, a rectangle was extracted from the workspace shown in Fig. 4. This rectangle ${ }^{2}$ has its base belonging to the interval $[-0.2675,1.3325]$ of the $\mathbf{x}_{\mathrm{b}}$-axis and its height belonging to the interval $[0.2$, 1.4] of the $\mathbf{y}_{\mathrm{b}}$-axis. In this test the robot is programmed to pass by 40 paths, with 40 points each one, from top to bottom on the rectangle, enumerated and aligned to the $\mathrm{x}_{\mathrm{b}}$ axis. Odd paths follow from left to right while even paths follow right to left. The first path has the start point, at the upper left corner of the rectangle (see Fig. 5).

Figure 5 shows the area of the swept rectangle inserted in the 4DOF redundant robot workspace detailing the path.

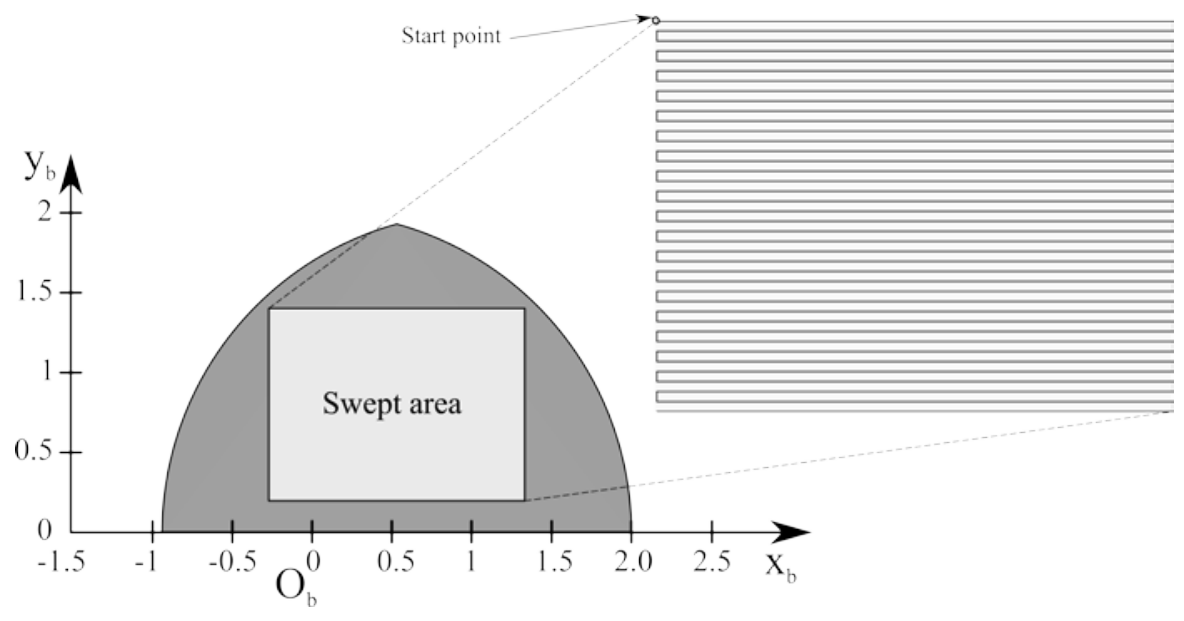

FIGURE 5. RECTANGULAR AREA WITHIN THE 4DOF WORKSPACE WITH DETAILED PATH.

Figures 6, 7, 8 and 9 show the computed values of the actuated-joint variables ${ }^{3} d_{1}, d_{3}, d_{4}$ and $d_{6}$, respectively, for sweeping the rectangle. Figure 10 shows the reached values for CI parameter at each point

\footnotetext{
${ }^{2}$ In all figures, the units of $\mathbf{x}_{\mathrm{b}}$ and $\mathbf{y}_{\mathrm{b}}$ axes are length units - l.u.

${ }^{3}$ The P-pair displacements units are presented in length units - l.u.
} 
in the rectangle, and Figs. 11 and 12 show the behavior of the computed coefficients $\tilde{f}_{4}$ and $\tilde{f}_{6}$ at each point of the rectangle.

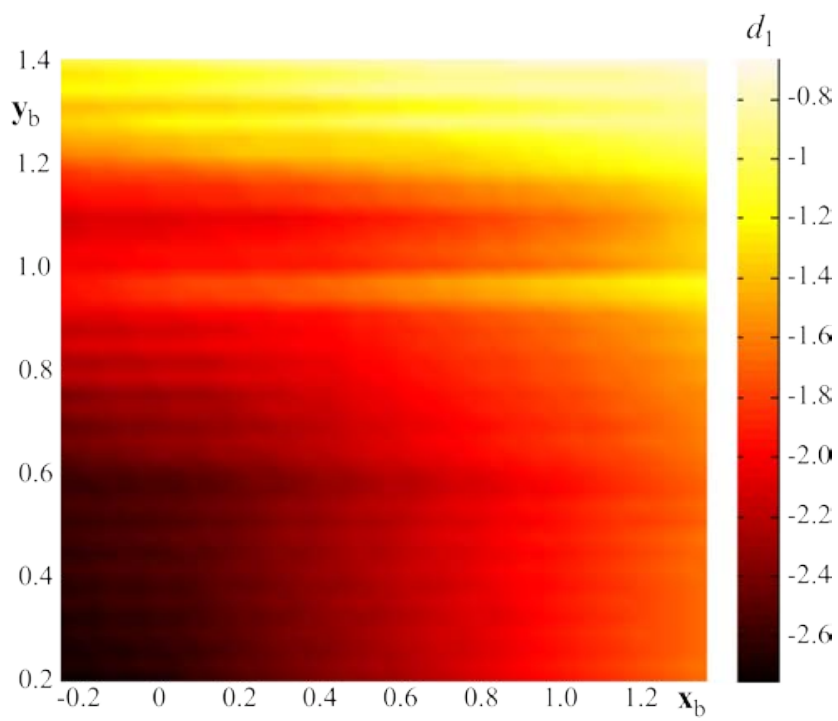

FIGURE 6. COMPUTED VALUES OF $d_{1}$ IN THE SWEEPING TEST.

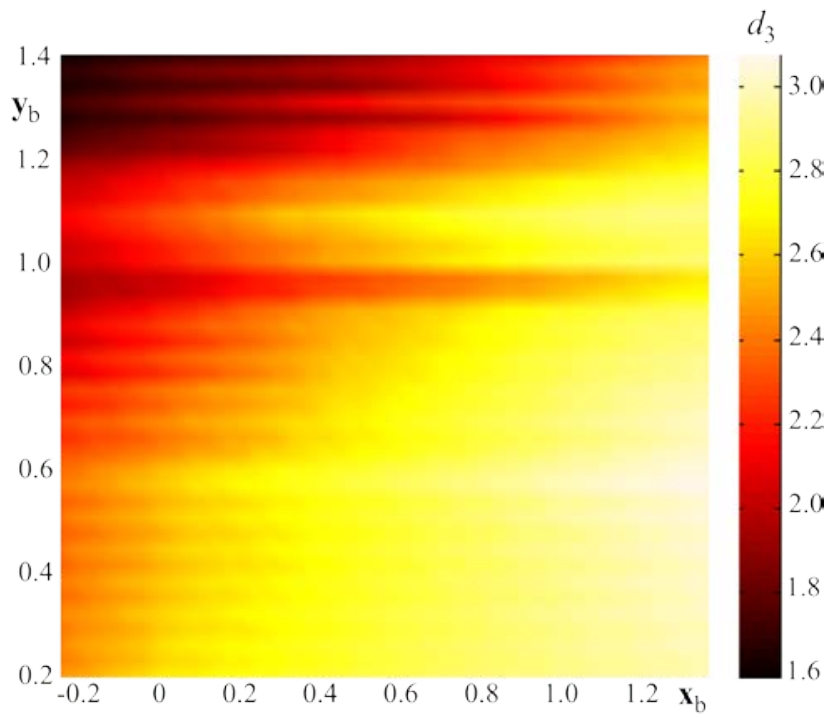

FIGURE 7. COMPUTED VALUES OF $d_{3}$ IN THE SWEEPING TEST. 


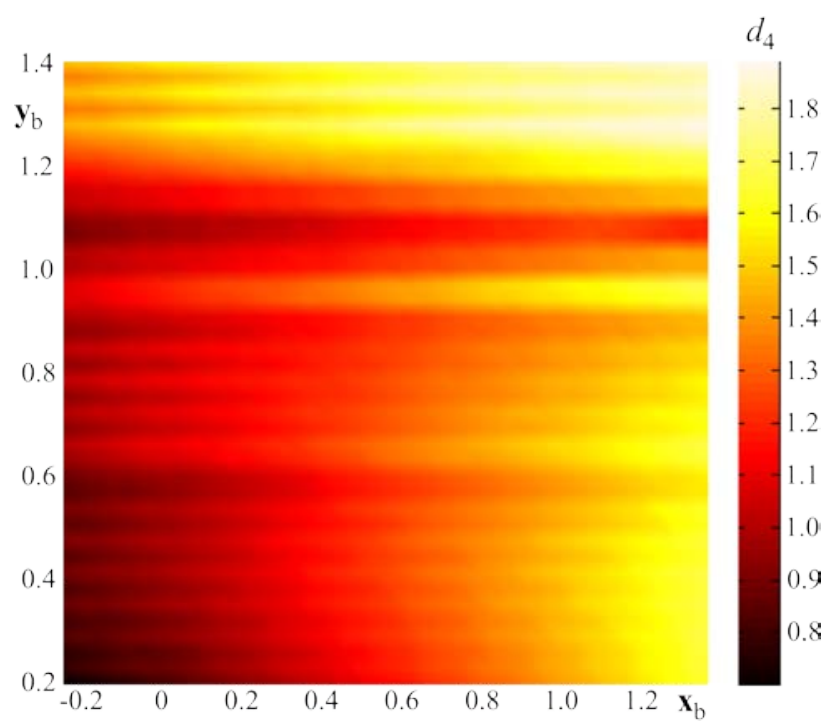

FIGURE 8. COMPUTED VALUES OF $d_{4}$ IN THE SWEEPING TEST.

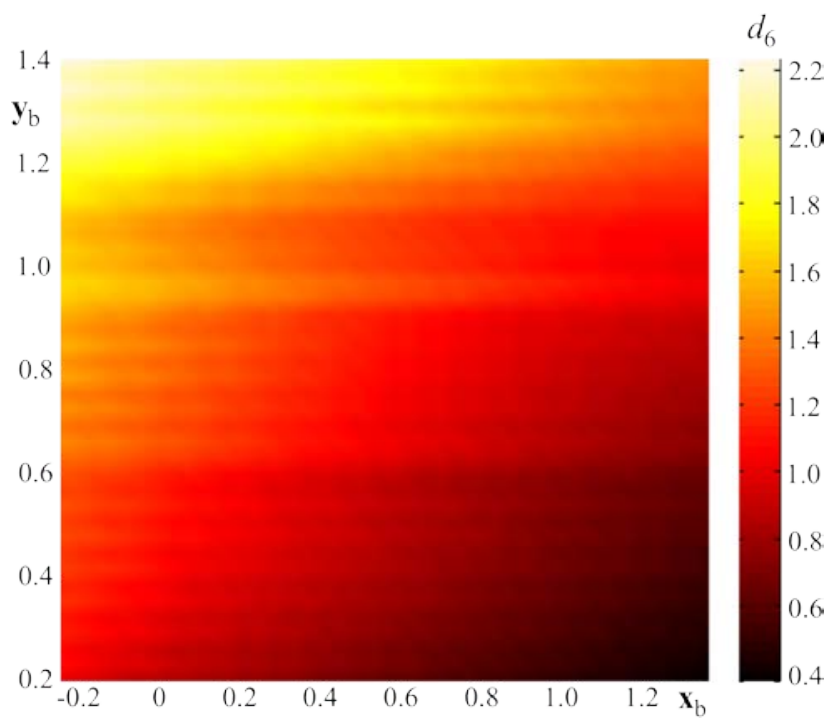

FIGURE 9. COMPUTED VALUES OF $d_{6}$ IN THE SWEEPING TEST. 


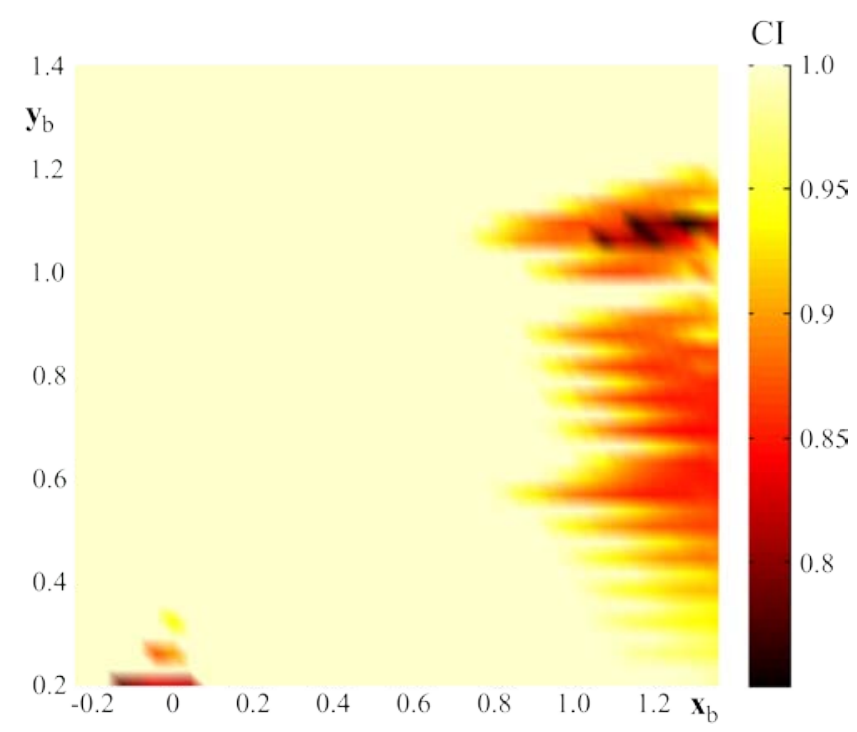

FIGURE 10. CI COMPUTED AT EACH POINT INSIDE THE RECTANGLE IN THE SWEEPING TEST

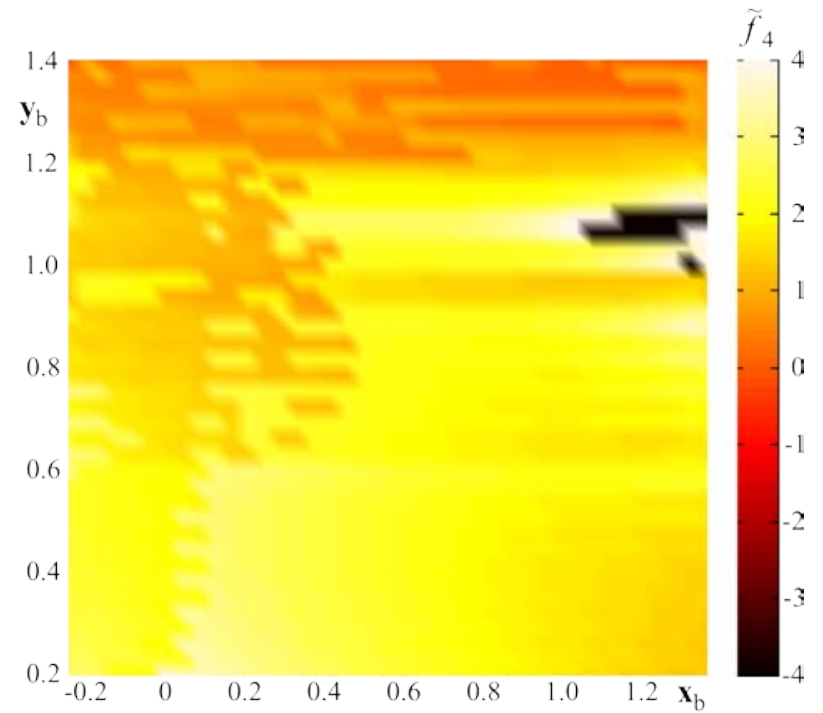

FIGURE 11. BEHAVIOR OF THE COEFFICIENT $\tilde{f}_{4}$ COMPUTED IN THE SWEPT RECTANGLE. 


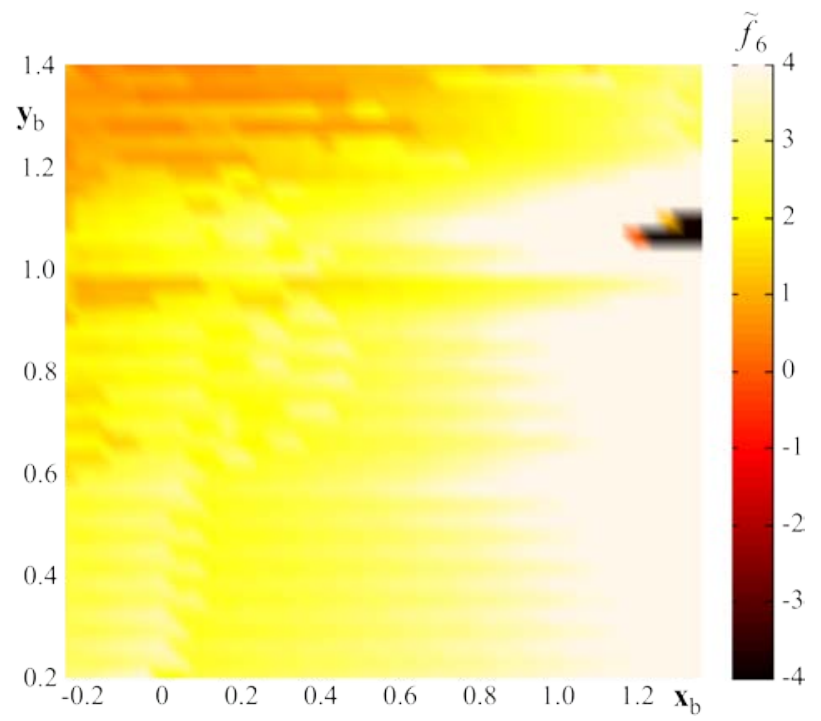

FIGURE 12. BEHAVIOR OF THE COEFFICIENT $\tilde{f}_{6}$ COMPUTED IN THE SWEPT RECTANGLE.

The analysis of these results reveals that the continuity in the displacements of the P-pairs is obtained, without relative abrupt variations in the position. Regarding the actuated joint rates, Table 1 shows the minimum and maximum computed values for each joint.

TABLE 1. MINIMUM AND MAXIMUM ACTUATED-JOINT RATES $\Delta T=1 \mathrm{~S}$ (TEST 1).

\begin{tabular}{|c|l|l|}
\hline Primary P-pair & Min. rate & Max. rate \\
\hline$d_{1}$ & -0.11544 1.u. $/ \Delta t$ & 0.07617 l.u. $/ \Delta t$ \\
\hline$d_{3}$ & -0.08094 1.u. $/ \Delta t$ & 0.07934 1.u. $/ \Delta t$ \\
\hline$d_{4}$ & -0.09953 l.u. $/ \Delta t$ & 0.11334 1.u. $/ \Delta t$ \\
\hline$d_{5}$ & -0.01595 l.u. $/ \Delta t$ & 0.01595 1.u. $/ \Delta t$ \\
\hline
\end{tabular}

The results for CI presented on Fig. 10, ranges from 0.75032 to 1.0 with the respective GCI=0.98313. Considering the values of $\mathrm{CI}$, it is interesting to note that the extended Jacobian remain far from singularities in practically all the swept area. It can be noted a decrease in values of CI near the left bottom vertex of the rectangle where the task was performed (see Fig. 10). In this region, the minimum value of CI is 0.75032 , which still is an acceptable value [32]. Anyway, it could be avoided by suitably planning the trajectories. 
Regarding to the behavior of the coefficients $\tilde{f}_{4}$ and $\tilde{f}_{6}$, both ranging from -4 to 4 , abrupt variations are observed, when performing the sweeping path, however, such variations do not reflect in significant variations in the displacement rates, as presented in the Table 1.

It is interesting to note that the coefficients $\tilde{f}_{4}$ and $\tilde{f}_{6}$ assumed their limit values in the regions where the lowest CI values occurred (see Figs 10, 11 and 12). This implies that an increase in the search range of the values for $\tilde{f}_{4}$ and $\tilde{f}_{6}$ allows to improve $\mathrm{CI}$ in such regions and consequently to improve GCI.

In this test it was spent the time of 18.255 s, or $11.25 \mathrm{~ms} /$ point.

\subsubsection{Circular path - Test 2}

The second test consists in making the end-effector track a sequence of turns in a closed circular path. The purpose of this study is to evaluate the joint displacements behavior regarding to the cyclicity problem.

In this test, 4000 points were selected on the 12 counterclockwise laps performed a circular path with radius $=0.5$ l.u. and center at the coordinates $(0.5325,0.8)$ l.u. Figure 13 shows the path for the test 2 .

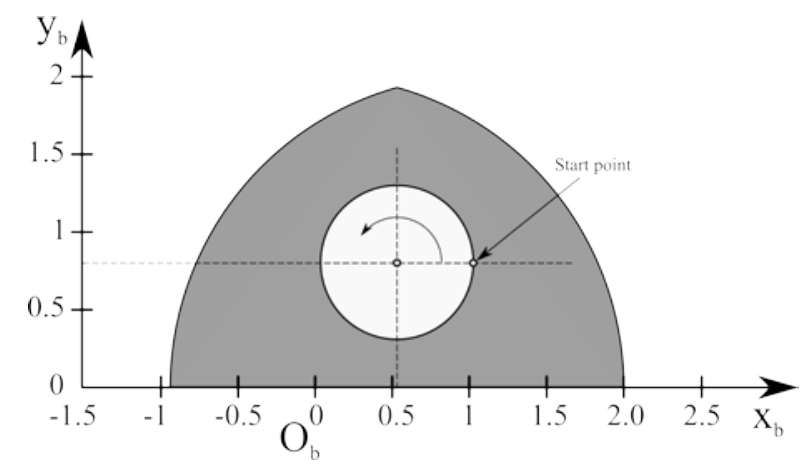

FIGURE 13. CIRCULAR PATH WITHIN THE 4DOF WORKSPACE.

Figures 14, 15, 16 and 17 show the computed values of the actuated-joint variables $d_{1}, d_{3}, d_{4}$ and $d_{6}$, respectively, and Figs 18 and 19 show the behavior of the computed coefficients $\tilde{f}_{4}$ and $\tilde{f}_{6}$, respectively. 


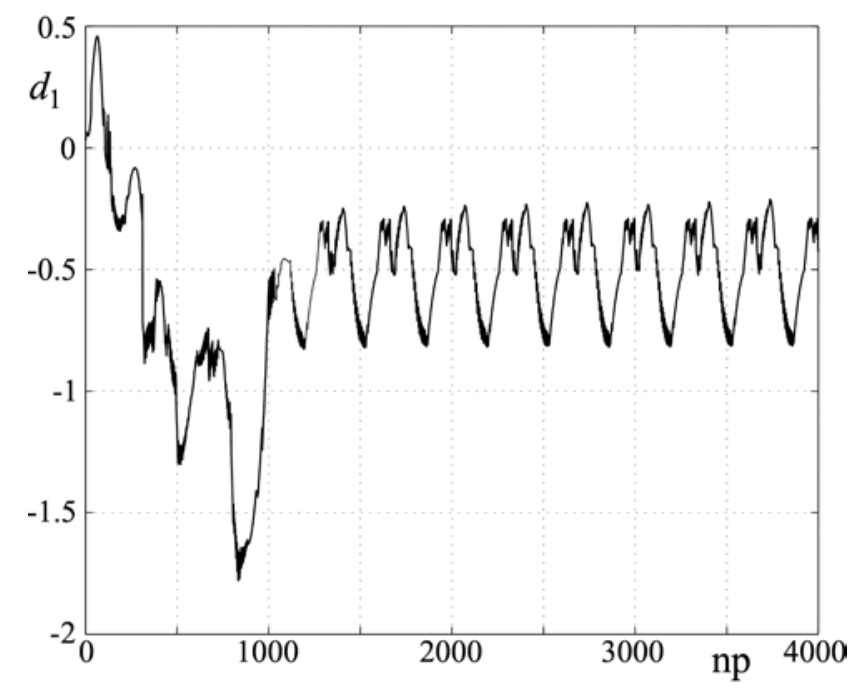

FIGURE 14. COMPUTED VALUES OF $d_{1}$ IN THE CIRCULAR TEST.

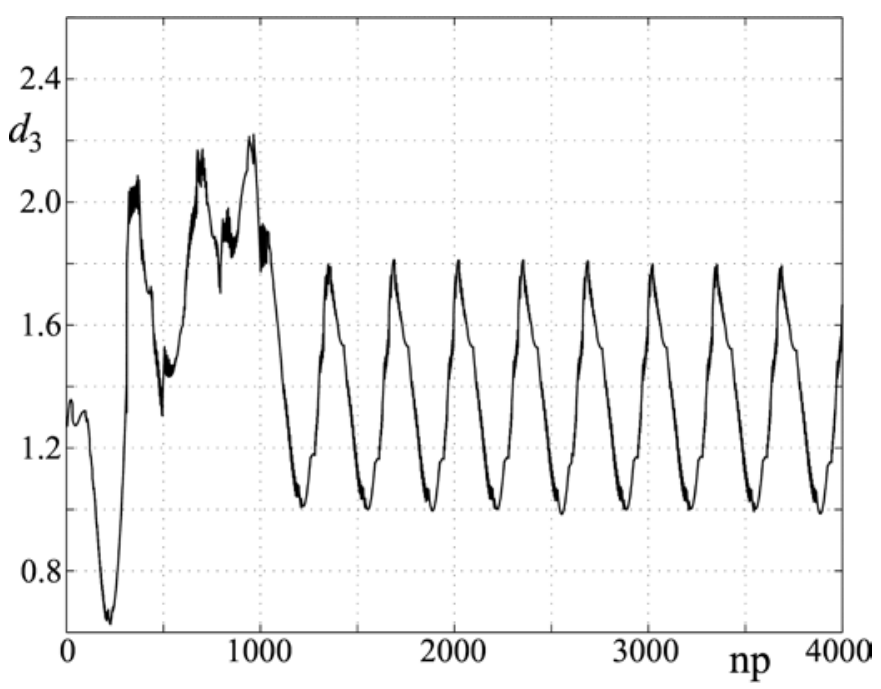

FIGURE 15. COMPUTED VALUES OF $d_{3}$ IN THE CIRCULAR TEST. 


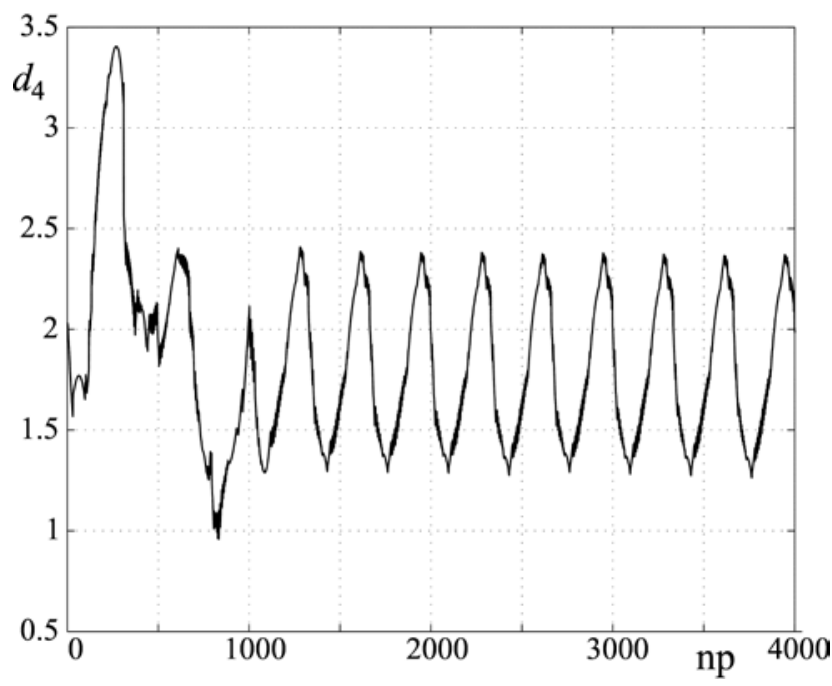

FIGURE 16. COMPUTED VALUES OF $d_{4}$ IN THE CIRCULAR TEST.

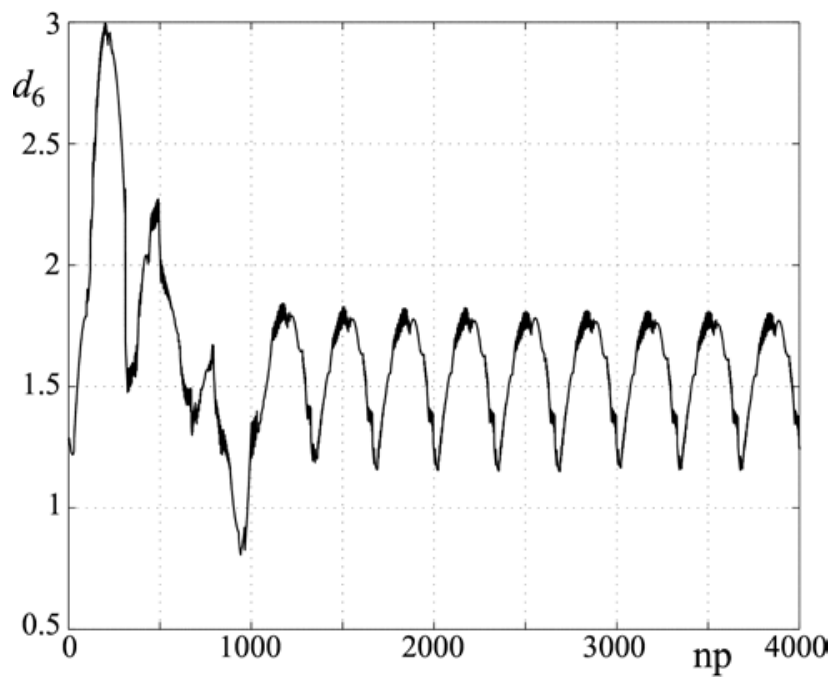

FIGURE 17. COMPUTED VALUES OF $d_{6}$ IN THE CIRCULAR TEST. 


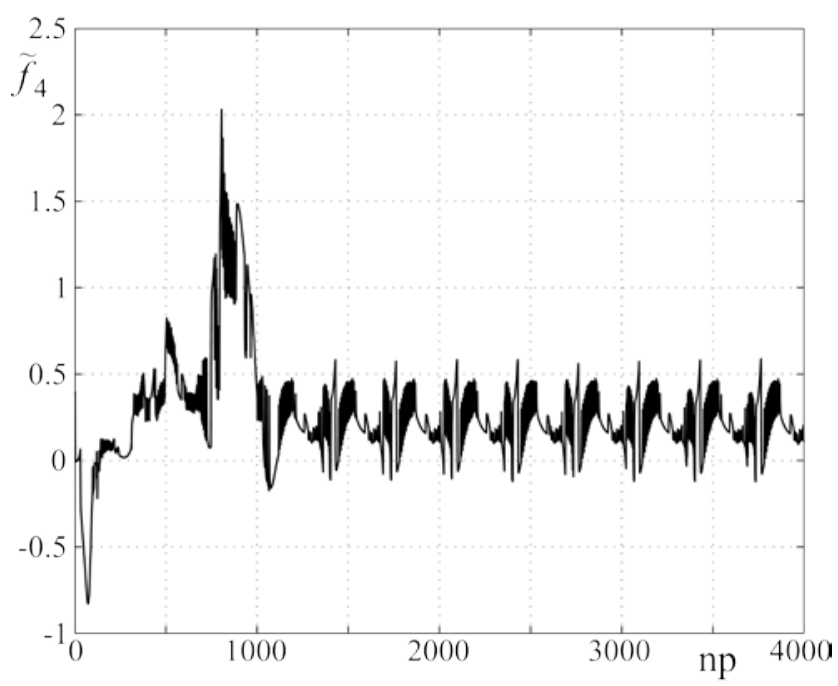

FIGURE 18. BEHAVIOR OF THE COEFFICIENT $\tilde{f}_{4}$ COMPUTED IN THE CIRCULAR TEST.

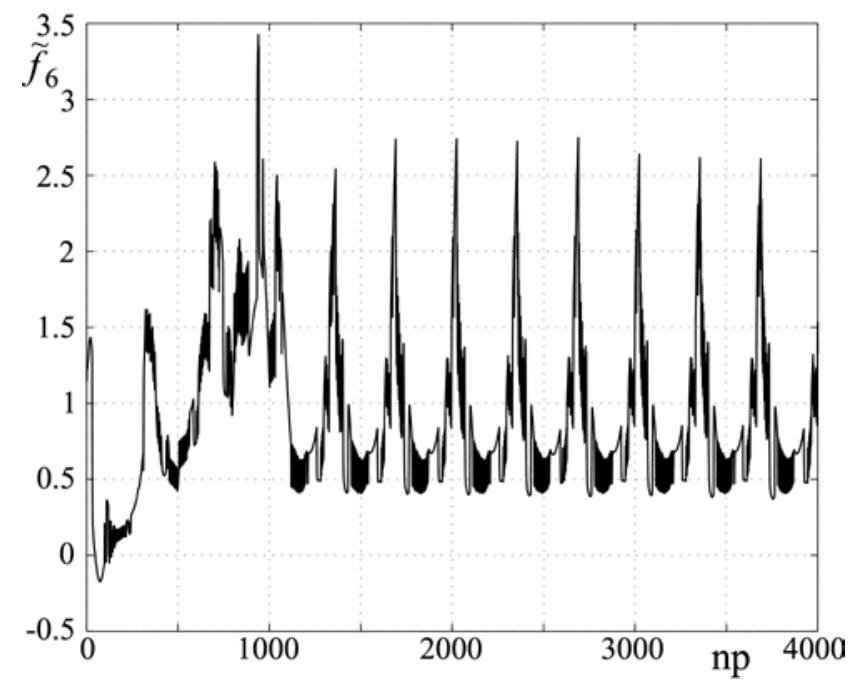

FIGURE 19. BEHAVIOR OF THE COEFFICIENT $\tilde{f}_{6}$ COMPUTED IN THE CIRCULAR TEST.

Regarding the computed values of the actuated-joint variables, it is observed that, in Figs. 14-19, the occurrence of an oscillating behavior in the variables is due to the fact that the end-effector coordinates oscillate for tracking a circular path. Also, it is observed that there is a transient interval in the amplitudes of these oscillations, between points 1 and 1000. After such transient, the displacements become harmonic with respect to each turn in the circular path; therefore, from the point 1000, the cyclicity problem vanishes.

In this test, $\mathrm{CI}$ ranges from 0.88324 to 1.0 , with $\mathrm{GCI}=0.99978$. These results demonstrate, as in test 1 , that the calculated values for the coefficients keep the robot far from singularities. 
Regarding the actuated-joint rates, Table 2 presents their maximum and minimum values. These values show that, despite the transients and some values higher when compared with values of test 1 (see Table 1), there were not significant rate variations in the joint positions with respect to the joint position magnitudes.

In this test, the coefficients ranged as $-0.82819<\tilde{f}_{4}<2.02987$ and $-0.17276<\tilde{f}_{6}<3.42573$ and a noisy behavior is observed, indicating a high variation of these values along the path (see Figs 18 and 19). Even so, the values computed for $\tilde{f}_{4}$ and $\tilde{f}_{6}$ at each point kept the redundant robot within suitable values for CI and joint displacements.

This test lasted 40.0366s, that is, $1.00 \mathrm{~ms} /$ point.

TABLE 2. MINIMUM AND MAXIMUM ACTUATED-JOINT RATES $\triangle T=1 \mathrm{~S}$ (TEST 2).

\begin{tabular}{|c|c|c|}
\hline Primary P-pair & Min. rate & Max. rate \\
\hline$d_{1}$ & -0.51549 l.u. $/ \Delta t$ & 0.19332 l.u. $/ \Delta t$ \\
\hline$d_{3}$ & -0.10200 l.u. $/ \Delta t$ & 0.48257 l.u. $/ \Delta t$ \\
\hline$d_{4}$ & -0.65603 l.u. $/ \Delta t$ & 0.20165 l.u. $/ \Delta t$ \\
\hline$d_{6}$ & -0.62690 l.u. $/ \Delta t$ & 0.16724 l.u. $/ \Delta t$ \\
\hline
\end{tabular}

\subsection{Time Evaluation}

As discussed, for general applications using the extended Jacobian, fixed values are used for the coefficients of the constraint functions. For comparison purposes, a complementary analysis was done for the tests 1 and 2 above developed, using for that, fixed values for $\tilde{f}_{4}$ and $\tilde{f}_{6}$. Recovering the results presented in the tests for the proposed method, Tables 3 and 4 comparatively present the computation times and the GCIs obtained for fixed and adaptive coefficients values for test 1 and test 2 respectively.

TABLE 3. COMPARATIVE PERFORMANCE VALUES TO THE TEST 1.

\begin{tabular}{|c|c|c|}
\cline { 2 - 3 } \multicolumn{1}{c|}{} & $\begin{array}{c}\text { Fixed values }^{4} \\
\tilde{f}_{4}=1 \text { and } \tilde{f}_{6}=-1\end{array}$ & $\begin{array}{c}\text { Adaptive values } \\
\text { (Proposed) }\end{array}$ \\
\hline Time/point & $0.4 \mathrm{~ms} /$ point & $11.25 \mathrm{~ms} /$ point \\
\hline GCI & 0.35276 & 0.98313 \\
\hline
\end{tabular}

${ }^{4}$ The values of the coefficients were obtained by varying the values within the intervals [-4, 4]. Increments of 0.5 were given for each coefficient until the best GCI was reached. 
TABLE 4. COMPARATIVE PERFORMANCE VALUES TO THE TEST 2.

\begin{tabular}{|c|c|c|}
\hline & $\begin{array}{l}\text { Fixed values } \\
\tilde{f}_{4}=1 \text { and } \tilde{f}_{6}=-1\end{array}$ & $\begin{array}{l}\text { Adaptive values } \\
\text { (Proposed) }\end{array}$ \\
\hline Time/point & $0.35 \mathrm{~ms} /$ point & $1.0 \mathrm{~ms} /$ point \\
\hline GCI & 0.30633 & 0.99978 \\
\hline
\end{tabular}

It is observed in this comparative analysis, that the time/point spent for calculations by the computer for fixed coefficients, varies (approximately) from 3 to 30 times faster when compared with the proposed adaptive adjustment algorithm for the coefficients. The difference in processing times is due to the fact that for fixed values, the search step is not performed, however, it is worth noting the improvement in GCI values, being increased 3 times to near 1 for both tests.

This comparison shows that the proposed adaptive algorithm improves the isotropic condition of the extended Jacobian, by moving it away and remaining it out of singular conditions. It is also worth mentioning that the fixed values for the coefficients were chosen empirically (aiming the best GCI) do not guarantee the avoidance of singular condition for all postures inside the workspace of the robot (see Fig. 1).

\section{CONCLUSION}

This paper presented a proposal to improve the kinematics models of redundant robots based on extended Jacobian. An algorithm has been proposed, aiming at continuously adjusting the coefficients of kinematic constraint functions, added as secondary tasks in the composition of the extended Jacobian. The adopted quality criterion of such adjustments, was the maximization of the conditioning index as function of the coefficients of the kinematic constraint functions, in each numerical iteration. The proposed algorithm is simple to be implemented, versatile and numerically stable, incorporating robustness in the solution of inverse kinematics by means of extended Jacobian.

Numerical tests were performed using a planar redundant robot. The results have showed the control over the conditioning index and the elimination of the cyclicity problem in the extended Jacobian. The proposed algorithm has its main application in offline task, or for online tasks, when the sampling time is greater than the calculation times per point, which in the studied case should be greater than $11.25 \mathrm{~ms}$ (see Table 3). 
The perspectives are to expand the proposed approach for spatial tasks and collision avoidance applications, as well as, improvements in the processing of the coefficients searching computations, in order to reduce the execution time of the algorithm, aiming at increasing the number of online applications. Other work perspectives include the analysis of the relationships between the proposed extended Jacobian and the existing classical methods such as nullspace-based strategies

\section{ACKNOWLEDGMENT}

This work has been developed at the Laboratory of Advanced Mechanics (MECH-LAV) of Ferrara Technopole, supported by UNIFE FAR2017 fund, by Regione Emilia Romagna (District Councillorship for Productive Assets, Economic Development, Telematic Plan) POR-FESR 2007-2013, Attività I.1.1, in partnership with Laboratory of Applied Robotics of Federal University of Santa Catarina, supported by CNPq - Conselho Nacional de Desenvolvimento Científico e Tecnológico (National Council for Scientific and Technological Development) Project 309241/2017-0, Brazil.

\section{REFERENCES}

[1] B. Siciliano, L. Sciavicco, L. Villani and G. Oriolo, "Modeling and control of robot manipulator", Springer. 2009.

[2] J. Baillieul, "Kinematic programming alternatives for redundant manipulators", Proceedings IEEE International Conference on Robotics and Automation, 1985.

[3] A. A. Campos Bonilla, R. Guenther and D. Martins, "Differential kinematics of parallel manipulators using Assur virtual chains”, Proceedings of the Institution of Mechanical Engineers. Part C, Journal of Mechanical Engineering Science, vol. 223, pp. 1697-1711, 2009

[4] C. Gosselin and J. Angeles, "Singularity analysis of closed-loop kinematic chains," in IEEE Transactions on Robotics and Automation, vol. 6, no. 3, pp. 281-290, Jun 1990.

[5] C. H. F. Santos, R. Guenther, D. Martins and E.R. De-Pieri, "Virtual kinematic chains to solve the underwater vehicle-manipulator systems redundancy”, Journal of the Brazilian Society of Mechanical Sciences and Engineering, vol. 28, no. 3, 2006. 
[6] H. Simas, R. Guenther, D. Da-Cruz and D. Martins, “A new method to solve robot inverse kinematics using Assur virtual chains”, Robotica Cambridge, vol. 27, no. 7, pp. 1017-1026, 2009.

[7] M. Šoch, and R. Lórencz. "Solving Inverse Kinematics - A New Approach to the Extended Jacobian Technique”, CORE, Czech Technical University, vol. 43, no. 2, 2005.

[8] C. A. Klein, C. Chu-Jenq and S. Ahmed, “A New Formulation of the Extended Jacobian Method and Its Use in Mapping Algorithmic Singularities for Kinematically Redundant Manipulators”, IEEE Transactions on Robotics and Automation, vol. 11, no. 1, pp. 50-55, 1995.

[9] D. P. Martin, J. Baillieul, and J. M. Hollerbach, "Resolution of Kinematic Redundancy Using Optimization Techniques", IEEE Transactions on Robotics and Automation, vol. 5, pp. 529-533, Aug. 1989.

[10] B. Siciliano, "Kinematic Control of Redundant Robot Manipulators: A Tutorial", Journal of Intelligent and Robotic Systems, vol. 3, no. 3, pp. 201-212, 1990.

[11] K. A. O'Neil, Y. Chen and J. Seng, "Removing singularities of resolved motion rate control of mechanisms, including self-motion", in IEEE Transactions on Robotics and Automation, vol. 13, no. 5, pp. 741-751, Oct 1997.

[12] D. E. Whitney, “The Mathematics of Coordinated Control of Prosthetic Arms and Manipulators”, ASME. J. Dyn. Sys. Meas. Control, vol 94, no 4, pp. 303-309, Dec 1972.

[13] G. Tevatia and S. Schaal, "Inverse kinematics for humanoid robots", Proceedings 2000 ICRA. Millennium Conference. IEEE International Conference on Robotics and Automation. Symposia Proceedings, vol 1, pp. 294-299, San Francisco, CA, 2000.

[14] G. Marani, J. Kim, J. Yuh and W. K. Chung, "A real-time approach for singularity avoidance in resolved motion rate control of robotic manipulators", Proceedings 2002 IEEE International Conference on Robotics and Automation, vol. 2, pp. 1973-1978, 2002.

[15] M. G. Marcos, J.A. Tenreiro Machado and T.-P. Azevedo-Perdicoúlis, “Trajectory planning of redundant manipulators using genetic algorithms”, Communications in Nonlinear Science and Numerical Simulation, vol. 14, no. 7, pp. 2858-2869, 2009. 
[16] C. Klein, C. Chu-Jenq and S. Ahmed, "Use of an extended Jacobian method to map algorithmic singularities", Proceedings IEEE International Conference on Robotics and Automation, Atlanta, vol. 3, pp. 632-637, 1993.

[17] J. Baillieul, "Avoiding obstacles and resolving kinematic redundancy," Proceedings IEEE International Conference on Robotics and Automation, pp. 1698-1704, 1986.

[18] K. Tchon, “Optimal Extended Jacobian Inverse Kinematics Algorithms for Robotic Manipulators”, IEEE Transactions on Robotics, vol. 24, no. 6, pp. 1440-1445, 2008.

[19] J. Ratajczak, "Design of inverse kinematics algorithms: extended Jacobian approximation of the dynamically consistent Jacobian inverse”, Archives of Control Sciences, vol. 25, no. 1, 2015.

[20] H. Simas, A, Dias and D. Martins, "Extended Jacobian for Redundant Robots Obtained from the Kinematics Constraints”, ABCM Symposium Series Mechatronic. $1^{\text {st }}$ ed.: ABCM, vol. 12, pp. 10051014, 2012.

[21] B. Siciliano, and O. Kathib, "Handbook of Robotics", Springer Handbook of Robotics , 2016.

[22] P. Chang, "A closed-form solution for the control of manipulators with kinematic redundancy", Proceedings. 1986 IEEE International Conference on Robotics and Automation, pp. 9-14, 1986.

[23] G. Marani, J. Kim, J. Yuh and W. K. Chung, "A real-time approach for singularity avoidance in resolved motion rate control of robotic manipulators", Proceedings 2002 IEEE International Conference on Robotics and Automation, vol. 2, pp. 1973-1978, 2002.

[24] G. Tevatia and S. Schaal, "Inverse kinematics for humanoid robots," Proceedings 2000 ICRA. Millennium Conference. IEEE International Conference on Robotics and Automation. Symposia Proceedings, San Francisco, vol. 1, pp. 294-299, 2000.

[25] J. Baillieul, "Avoiding obstacles and resolving kinematic redundancy", Proceedings. IEEE International Conference on Robotics and Automation, pp. 1698-1704, 1986.

[26] L.-W Tsai, "Mechanism Design: Enumeration of Kinematic Structures According to Function”, Mechanical Engineering series, CRC Press, Washington, D.C., 2001.

[27] S. Chiaverini, "Singularity-robust task-priority redundancy resolution for real-time kinematic control of robot manipulators", in IEEE Transactions on Robotics and Automation, vol. 13, no. 3, pp. 398410, Jun 1997. 
[28] K. Tchoń and J. Jakubiak, “Extended jacobian motion planning algorithm for mobile manipulators”, IFAC Proceedings Volumes, vol. 36, no. 17 pp. 147-152, 2003.

[29] G. Marani, J. Kim, J. Yuh and W. K. Chung, "Algorithmic singularities avoidance in task-priority based controller for redundant manipulators", Proceedings 2003 IEEE/RSJ International Conference on Intelligent Robots and Systems (IROS 2003), vol. 3, pp. 3570-3574, 2003.

[30] C. D. Meyer, “Matrix analysis and applied linear algebra”, Society for Industrial and Applied Mathematics, Philadelphia-USA, 2000.

[31] C. Gosselin, J. Angeles, “A global performance index for the kinematic optimization of robotic manipulators,” Journal of Mechanical Design, 113 (3), 1991, pp. 220-226

[32] H, Simas H and R. Di Gregorio “Geometric Error Effects on Manipulators' Positioning Precision: A General Analysis and Evaluation Method”, ASME. J. Mechanisms Robotics, vol. 8, no. 6, 061016061016-10. doi:10.1115/1.4034577, 2016.

[33] C. Wampler, "Inverse kinematic functions for redundant manipulators," Proceedings. 1987 IEEE International Conference on Robotics and Automation, Raleigh, NC, USA, 1987, pp. 610-617. doi: 10.1109/ROBOT.1987.1087950

[34] J. Angeles, “The Design of Isotropic Manipulator Architectures in the Presence of Redundancies”, The International Journal of Robotics Research, 11(3), 196-201. doi:10.1177/027836499201100303

[35] S B. Nokleby and R. P. Podhorodeski, "Reciprocity-based resolution of velocity degeneracies (singularities) for redundant manipulators”, Mechanism and Machine Theory, vol. 36, no. 3, pp. 397409, 2001.

[36] I. Zaplana and L. Basanez, “A Novel Closed-Form Solution for the Inverse Kinematics of Redundant Manipulators through Workspace Analysis.” Mechanism and Machine Theory, vol. 121, pp. 829-843, 2018

[37] V. Kumar, S. Sen, S.S. Roy, S.K Das and S.N. Shome, "Inverse Kinematics of Redundant Manipulator using Interval Newton Method", IJEM, vol.5, no.2, pp.19-29, 2015.

[38] H. Toshani, and M. Farrokhi. "Real-Time Inverse Kinematics of Redundant Manipulators Using Neural Networks and Quadratic Programming: A Lyapunov-Based Approach.” Robotics and Autonomous Systems, vol. 62, no. 6, pp. 766-781, 2014. 
[39] C. Faria, F. Ferreira, W. Erlhagen, S. Monteiro and E. Bicho, "Position-Based Kinematics for 7-DoF Serial Manipulators with Global Configuration Control, Joint Limit and Singularity Avoidance.” Mechanism and Machine Theory, vol. 121, pp. 317-334, 2018.

[40] F. Blanchini, G. Fenu, G. Giordano and A. Pellegrino, “A Convex Programming Approach to the Inverse Kinematics Problem for Manipulators under Constraints.” European Journal of Control, vol. 33, pp. 11-23, 2017.

[41] V. V. M., J. S. Chembuly, and H. K. Voruganti. “An Efficient Approach for Inverse Kinematics and Redundancy Resolution Scheme of Hyper-Redundant Manipulators.” Advances in Mechanical Design, Materials and Manufacture, pp. 020019-1-020019-8, 2018.

[42] L. Huo and L. Baron. "The Self-Adaptation of Weights for Joint-Limits and Singularity Avoidances of Functionally Redundant Robotic-Task.” Robotics and Computer-Integrated Manufacturing, vol. 27, no. 2, pp. 367-376, 2011.

[43] D. Corinaldi, J. Angeles and M. Callegari, "Posture Optimization of a Functionally Redundant Parallel Robot.” Advances in Robot Kinematics 2016 Springer Proceedings in Advanced Robotics, pp. 101108, 2017.

[44] L. Huo and L. Baron. "Inverse Kinematics of Functionally-Redundant Serial Manipulators under Joint Limits and Singularity Avoidance.” Proceedings of the Int. Conference on Systems and Control, pp. 1-7, Marrakesh-Morocco, May 2007.

[45] J. Angeles, F. Ranjbaran and R.V. Patel, "On the Design of the Kinematic Structure of Seven-Axes Redundant Manipulators for Maximum Conditioning.” Proceedings 1992 IEEE International Conference on Robotics and Automation, May 1992.

[46] L. Jérémie. “Condition-Number Minimization for Functionally Redundant Serial Manipulators”, Master Thesis, McGill University, 2014.

[47] S. Yahya, M. Moghavvemi, H.A.F. Mohamed, “Geometrical Approach of Planar Hyper-Redundant Manipulators: Inverse Kinematics, Path Planning and Workspace.” Simulation Modelling Practice and Theory, vol. 19, no. 1, pp. 406-422, 2011. 
[48] D. Bertram, J. Kuffner, R. Dillmann and T. Asfour, "An integrated approach to inverse kinematics and path planning for redundant manipulators," Proceedings 2006 IEEE International Conference on Robotics and Automation, 2006. ICRA 2006., Orlando, FL, pp. 1874-1879, 2006.

[49] S. Kumar, S., S. Sukanavam and R. Balasubramanian, “An optimization approach to solve the inverse kinematics of redundant manipulator”, International Journal of Information and Systems Sciences, vol. 6, no. 4, pp. 414-423, 2010.

[50] H. Ananthanarayanan and R. Ordóñez, "Real-Time Inverse Kinematics of (2n+1)DOF HyperRedundant Manipulator Arm via a Combined Numerical and Analytical Approach”, Mechanism and Machine Theory, vol. 91, pp. 209-226, 2015.

[51] C. A. Klein and B. E. Blaho, "Dexterity measures for the design and control of kinematically redundant manipulators” Int. J. Rob. Res, vol. 6, no. 2, 72-83, July 1987.

[52] Y. Wei, S. Jian, S. He and Z. Wang, “General Approach for Inverse Kinematics of nR Robots.” Mechanism and Machine Theory, vol. 75, pp. 97-106, 2014. 


\section{List of Table Captions}

Table 1: MINIMUM AND MAXIMUM ACTUATED-JOINT RATES $\Delta \mathrm{T}=1 \mathrm{~s}$ (TEST 1).

Table 2: MINIMUM AND MAXIMUM ACTUATED-JOINT RATES $\Delta \mathrm{T}=1 \mathrm{~s}$ (TEST 2).

Table 3: COMPARATIVE PERFORMANCE VALUES TO THE TEST 1.

Table 4: COMPARATIVE PERFORMANCE VALUES TO THE TEST 2. 


\section{List of Figure Captions}

Figure 1: GEOMETRIC BEHAVIOR OF THE CONDITIONING INDEX AS A FUNCTION OF THE PARAMETERS $\tilde{f}_{4}$ AND $\tilde{f}_{6}$.

Figure 2: FLOWCHART OF THE PROPOSED ALGORITHM.

Figure 3: PLANAR 4DOF REDUNDANT ROBOT.

Figure 4: 4DOF WORKSPACE FOR $d_{1}=1.065 d_{4}=0$ WITH $0<\left\{d_{3}, d_{5}\right\}<2$.

Figure 5: RECTANGULAR AREA WITHIN THE 4DOF WORKSPACE WITH DETAILED PATH.

Figure 6: COMPUTED VALUES OF $d_{1}$ IN THE SWEEPING TEST.

Figure 7: COMPUTED VALUES OF $d_{3}$ IN THE SWEEPING TEST.

Figure 8: COMPUTED VALUES OF $d_{4}$ IN THE SWEEPING TEST.

Figure 9: COMPUTED VALUES OF $d_{6}$ IN THE SWEEPING TEST.

Figure 10: CI COMPUTED FOR EACH POINT INSIDE THE RECTANGLE IN THE SWEEPING TEST.

Figure 11: BEHAVIOR OF THE COEFFICIENT $\tilde{f}_{4}$ COMPUTED IN THE SWEPT RECTANGLE.

Figure 12: BEHAVIOR OF THE COEFFICIENT $\tilde{f}_{6}$ COMPUTED IN THE SWEPT RECTANGLE.

Figure 13: CIRCULAR PATH WITHIN THE 4DOF WORKSPACE.

Figure 14: COMPUTED VALUES OF $d_{1}$ IN THE CIRCULAR TEST.

Figure 15: COMPUTED VALUES OF $d_{3}$ IN THE CIRCULAR TEST.

Figure 16: COMPUTED VALUES OF $d_{4}$ IN THE CIRCULAR TEST.

Figure 17: COMPUTED VALUES OF $d_{6}$ IN THE CIRCULAR TEST.

Figure 18: BEHAVIOR OF THE COEFFICIENT $\tilde{f}_{4}$ COMPUTED IN THE CIRCULAR TEST.

Figure 19: BEHAVIOR OF THE COEFFICIENT $\tilde{f}_{6}$ COMPUTED IN THE CIRCULAR TEST. 
TABLE 1. MINIMUM AND MAXIMUM ACTUATED-JOINT RATES $\Delta T=1 \mathrm{~S}$ (TEST 1).

\begin{tabular}{|c|l|l|}
\hline Primary P-pair & Min. rate & Max. rate \\
\hline$d_{1}$ & -0.11544 1.u. $/ \Delta t$ & 0.07617 1.u. $/ \Delta t$ \\
\hline$d_{3}$ & -0.08094 l.u. $/ \Delta t$ & 0.07934 1.u. $/ \Delta t$ \\
\hline$d_{4}$ & -0.09953 l.u. $/ \Delta t$ & 0.11334 l.u. $/ \Delta t$ \\
\hline$d_{5}$ & -0.01595 l.u. $/ \Delta t$ & 0.01595 l.u. $/ \Delta t$ \\
\hline
\end{tabular}


TABLE 2. MINIMUM AND MAXIMUM ACTUATED-JOINT RATES $\Delta T=1 \mathrm{~S}$ (TEST 2).

\begin{tabular}{|c|c|c|}
\hline Primary P-pair & Min. rate & Max. rate \\
\hline$d_{1}$ & -0.51549 l.u. $/ \Delta t$ & 0.19332 l.u. $/ \Delta t$ \\
\hline$d_{3}$ & -0.10200 l.u. $/ \Delta t$ & 0.48257 l.u. $/ \Delta t$ \\
\hline$d_{4}$ & -0.65603 l.u. $/ \Delta t$ & 0.20165 l.u. $/ \Delta t$ \\
\hline$d_{6}$ & -0.62690 l.u. $/ \Delta t$ & 0.16724 l.u. $/ \Delta t$ \\
\hline
\end{tabular}


TABLE 3. COMPARATIVE PERFORMANCE VALUES TO THE TEST 1.

\begin{tabular}{|c|c|c|}
\cline { 2 - 3 } \multicolumn{1}{c|}{} & $\begin{array}{c}\text { Fixed values }^{5} \\
\tilde{f}_{4}=1 \text { and } \tilde{f}_{6}=-1\end{array}$ & $\begin{array}{c}\text { Adaptive values } \\
\text { (Proposed) }\end{array}$ \\
\hline Time/point & $0.4 \mathrm{~ms} /$ point & $11.25 \mathrm{~ms} /$ point \\
\hline GCI & 0.35276 & 0.98313 \\
\hline
\end{tabular}

${ }^{5}$ These values were obtained empirically by trial-and-error aiming to maximize GCI. 
TABLE 4. COMPARATIVE PERFORMANCE VALUES TO THE TEST 2.

\begin{tabular}{|c|c|c|}
\hline & Fixed values & \multirow{2}{*}{$\begin{array}{l}\text { Adaptive values } \\
\text { (Proposed) }\end{array}$} \\
\hline & $\tilde{f}_{4}=1$ and $\tilde{f}_{6}=-1$ & \\
\hline Time/point & $0.35 \mathrm{~ms} /$ point & $1.0 \mathrm{~ms} /$ point \\
\hline GCI & 0.30633 & 0.99978 \\
\hline
\end{tabular}




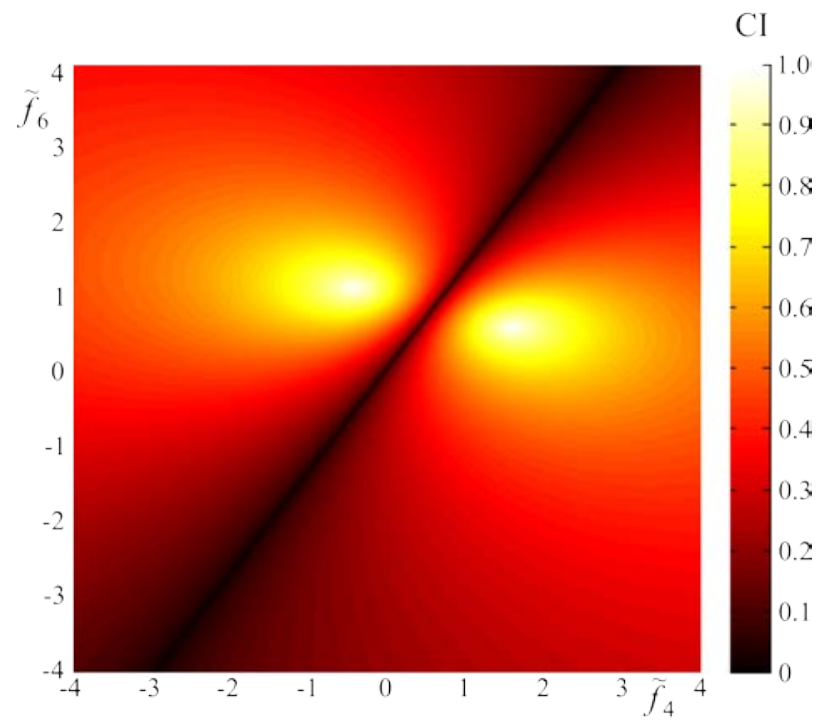

FIGURE 1. GEOMETRIC BEHAVIOR OF THE CONDITIONING INDEX AS A FUNCTION OF THE PARAMETERS $\tilde{f}_{4}$ AND $\tilde{f}_{6}$. 


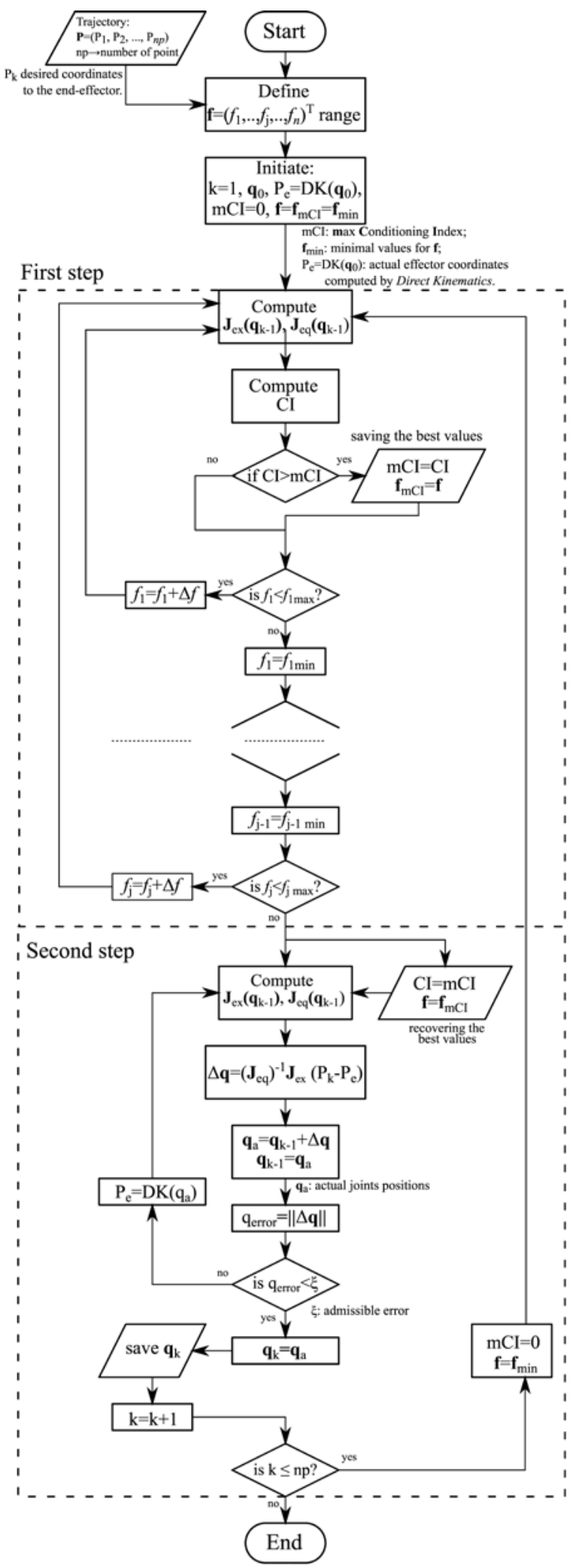

FIGURE 2. FLOWCHART OF THE PROPOSED ALGORITHM. 


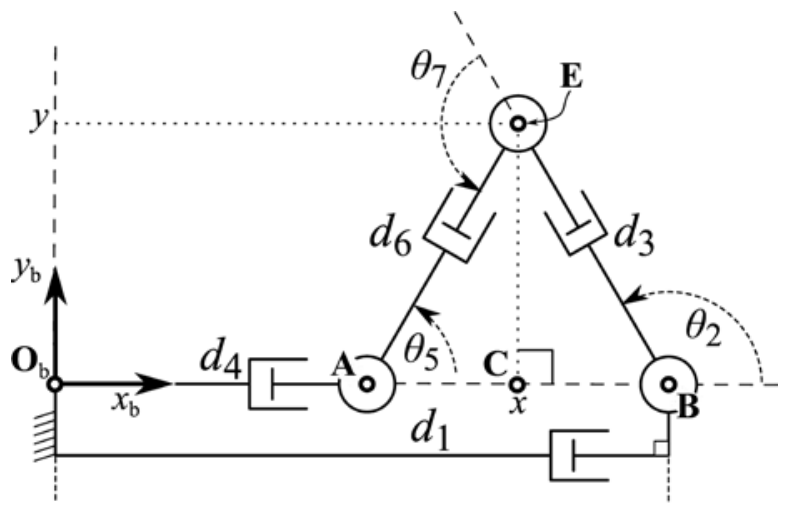

FIGURE 3. PLANAR 4DOF REDUNDANT ROBOT. 


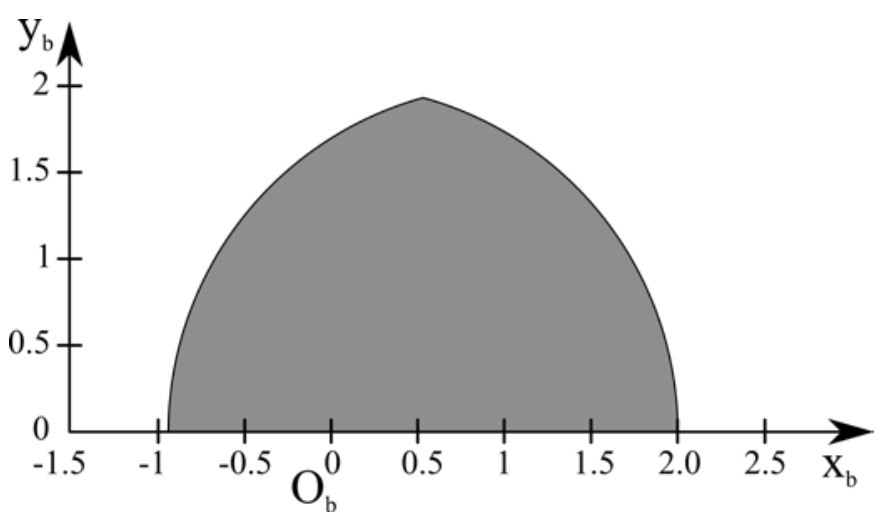

FIGURE 4. 4DOF WORKSPACE FOR $d_{1}=1.065 d_{4}=0$ WITH $0<\left\{d_{3}, d_{5}\right\}<2$. 


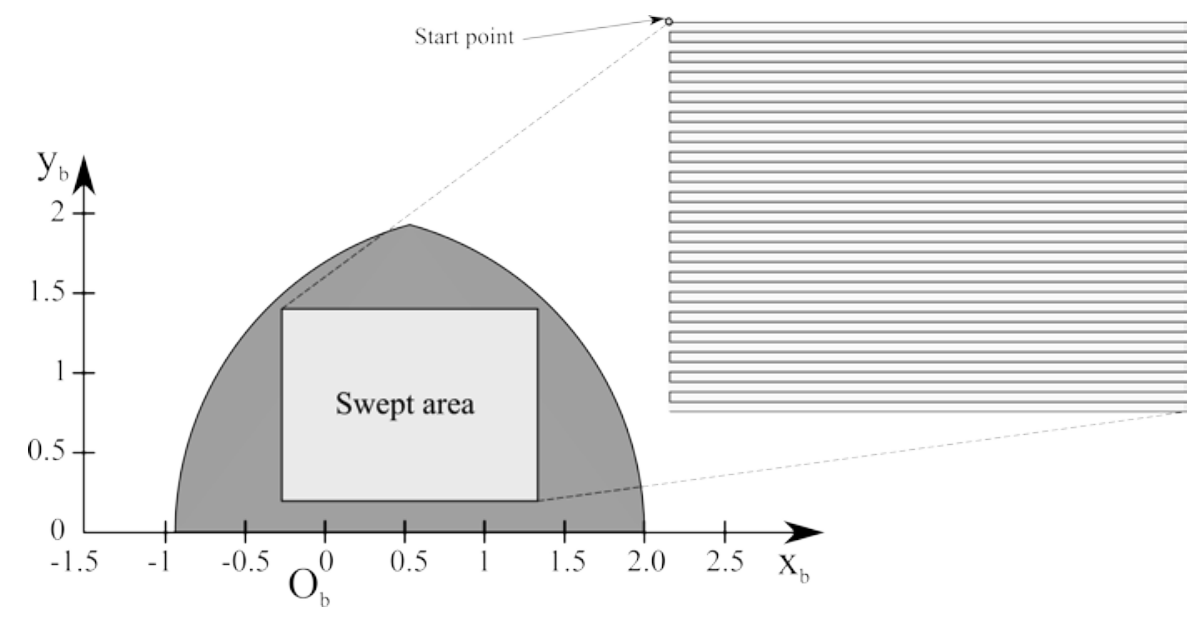

FIGURE 5. RECTANGULAR AREA WITHIN THE 4DOF WORKSPACE WITH DETAILED PATH. 


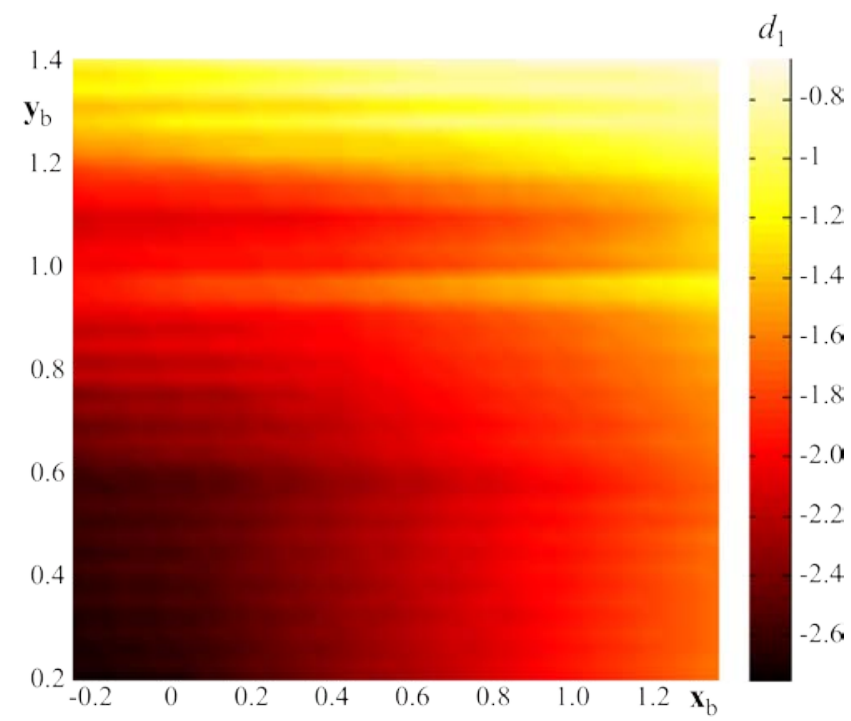

FIGURE 6. COMPUTED VALUES OF $d_{1}$ IN THE SWEEPING TEST. 


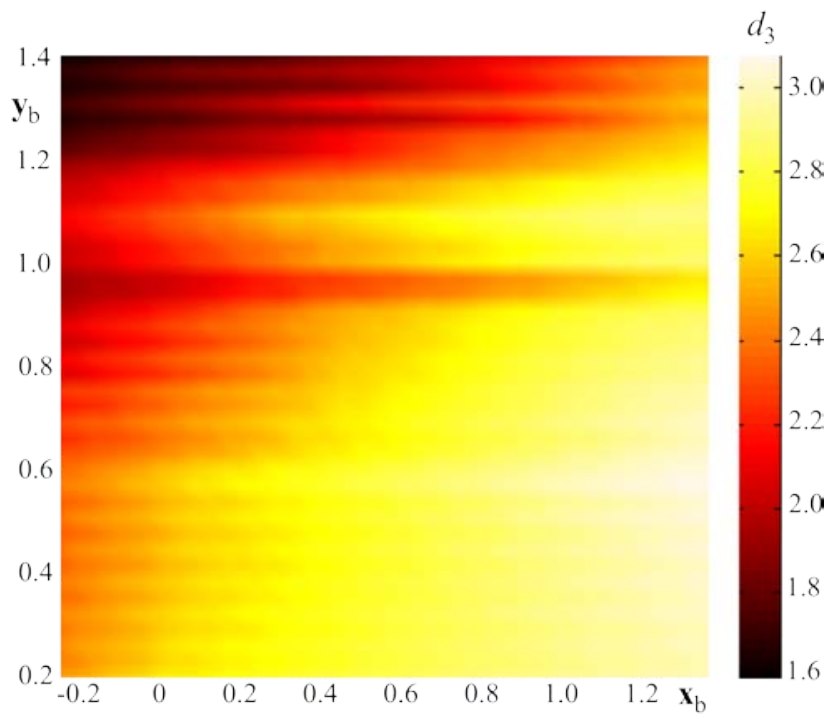

FIGURE 7. COMPUTED VALUES OF $d_{3}$ IN THE SWEEPING TEST. 


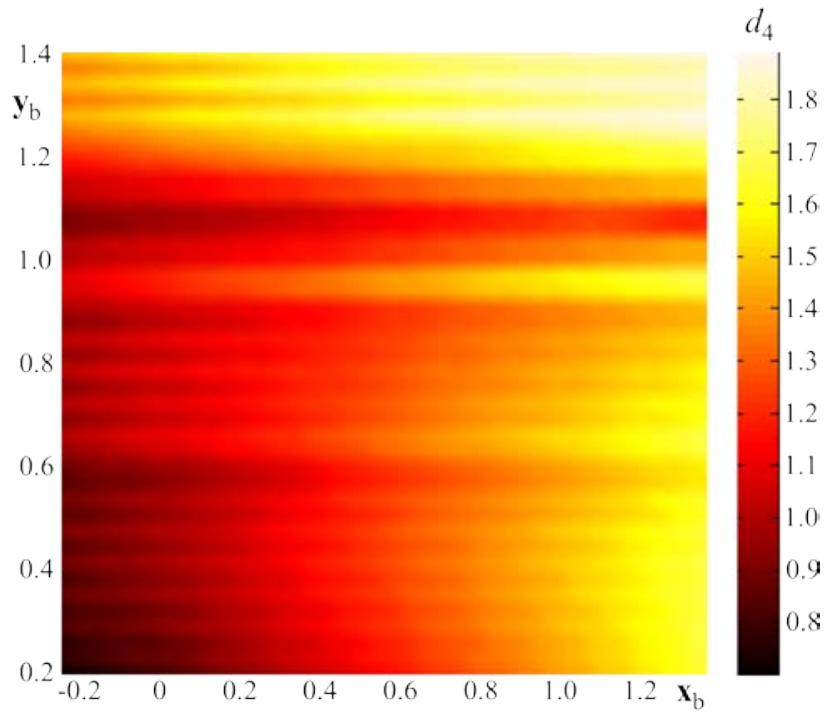

FIGURE 8. COMPUTED VALUES OF $d_{4}$ IN THE SWEEPING TEST. 


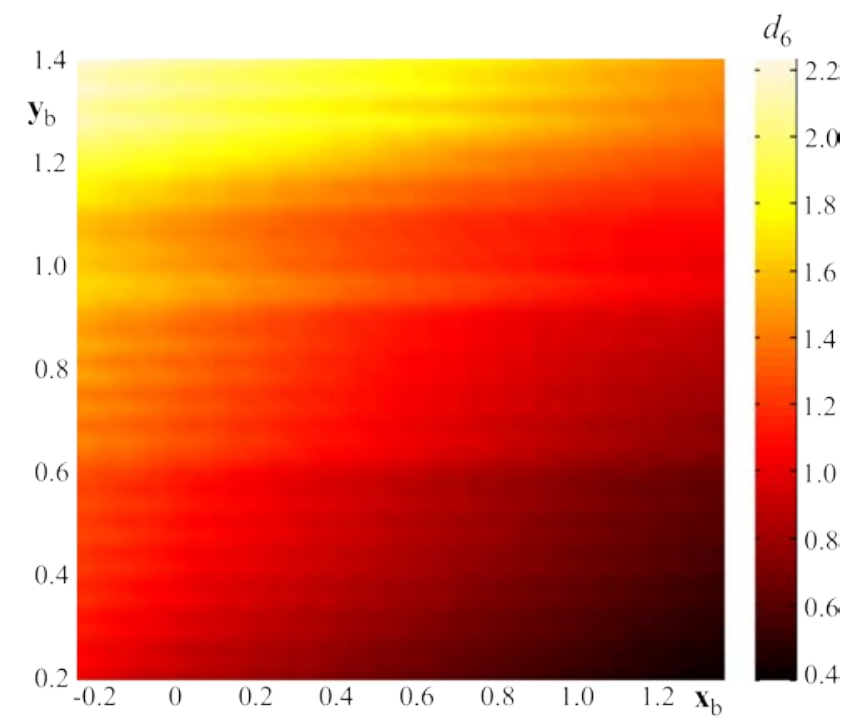

FIGURE 9. COMPUTED VALUES OF $d_{6}$ IN THE SWEEPING TEST. 


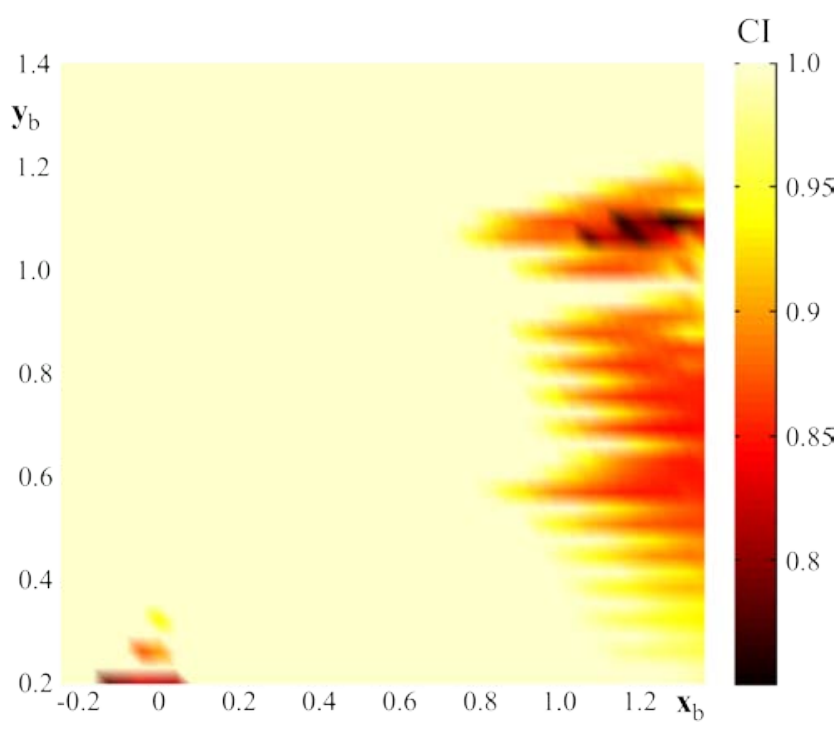

FIGURE 10. CI COMPUTED FOR EACH POINT INSIDE THE RECTANGLE IN THE SWEEPING TEST 


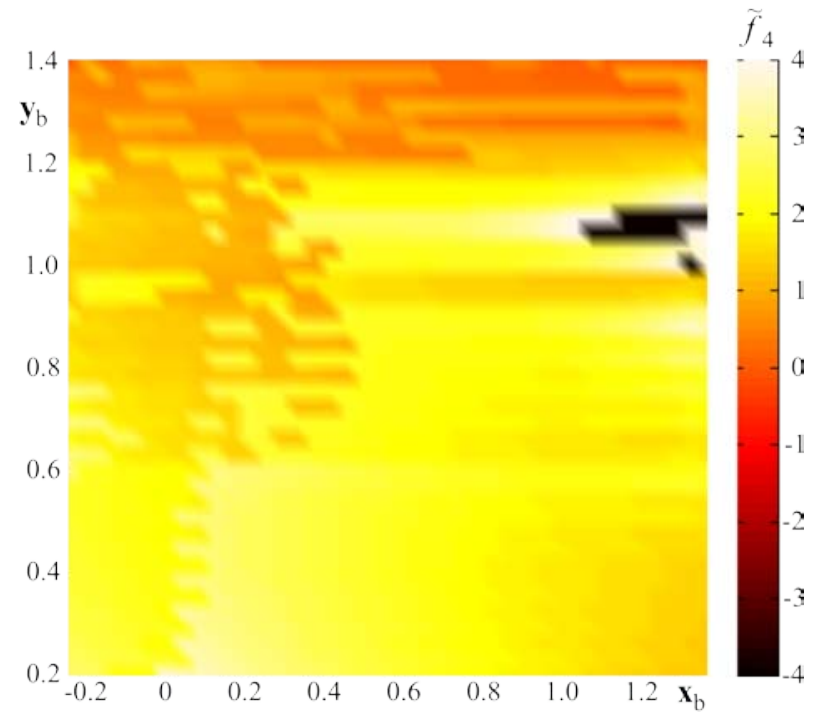

FIGURE 11. BEHAVIOR OF THE COEFFICIENT $\tilde{f}_{4}$ COMPUTED IN THE SWEPT RECTANGLE. 


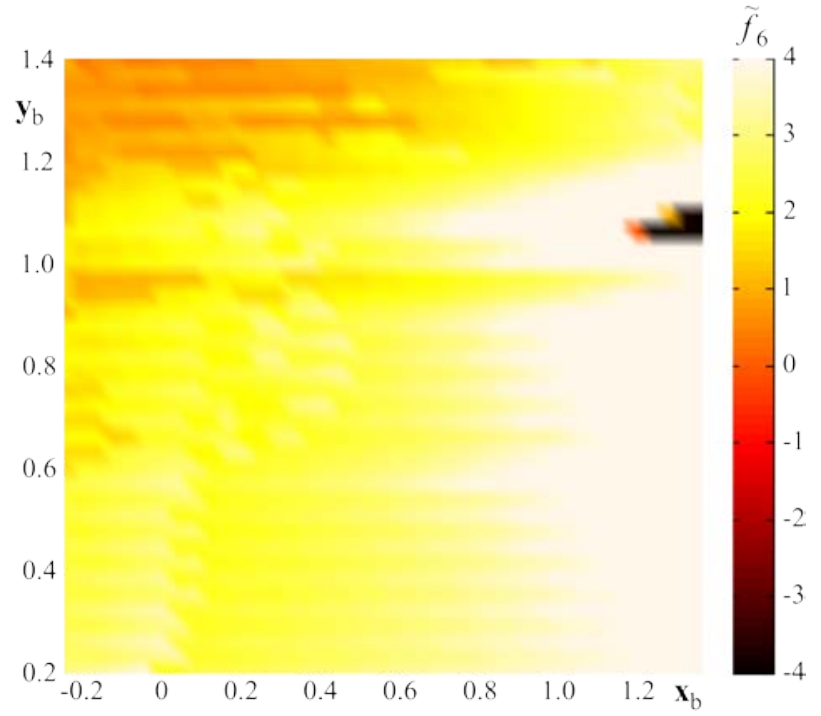

FIGURE 12. BEHAVIOR OF THE COEFFICIENT $\tilde{f}_{6}$ COMPUTED IN THE SWEPT RECTANGLE. 


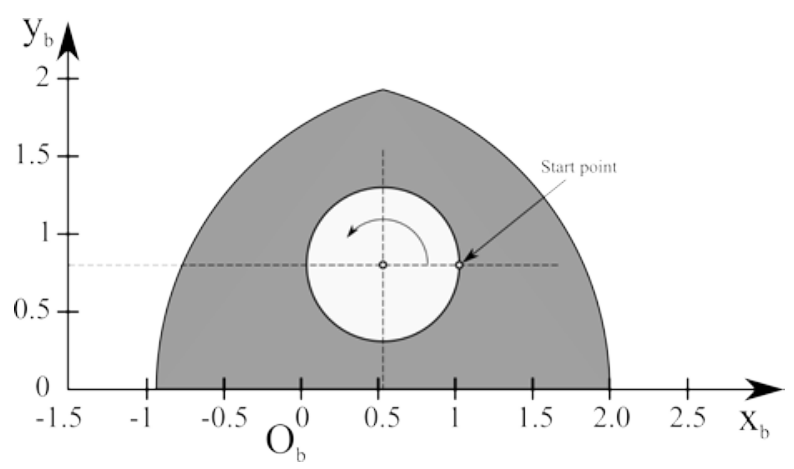

FIGURE 13. CIRCULAR PATH WITHIN THE 4DOF WORKSPACE. 


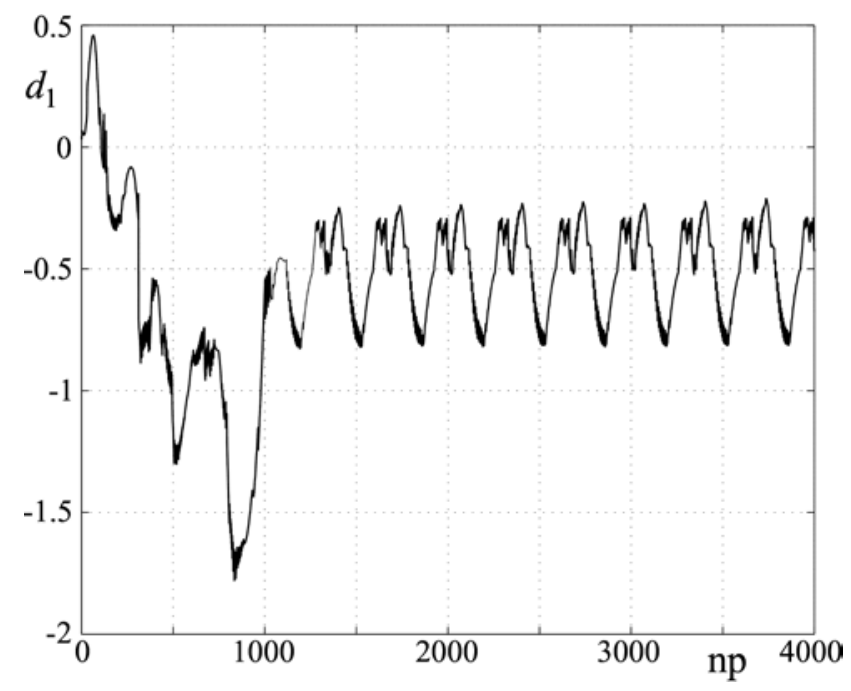

FIGURE 14. COMPUTED VALUES OF $d_{1}$ IN THE CIRCULAR TEST. 


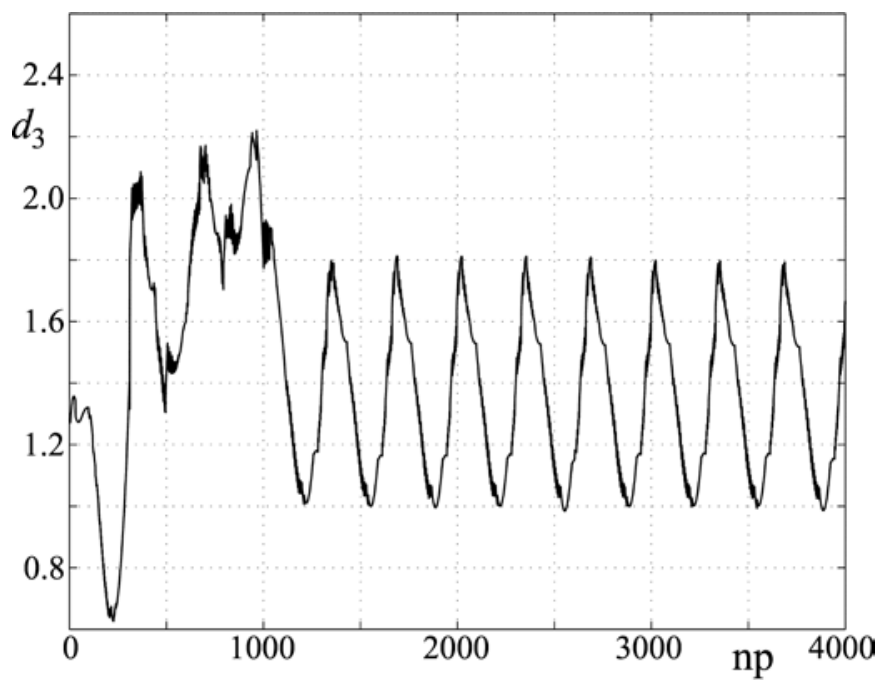

FIGURE 15. COMPUTED VALUES OF $d_{3}$ IN THE CIRCULAR TEST. 


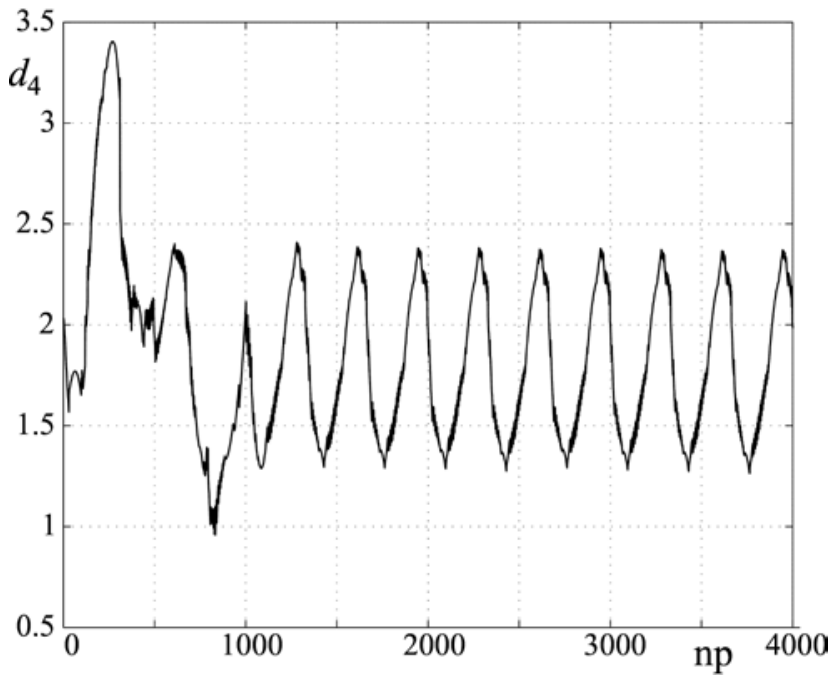

FIGURE 16. COMPUTED VALUES OF $d_{4}$ IN THE CIRCULAR TEST. 


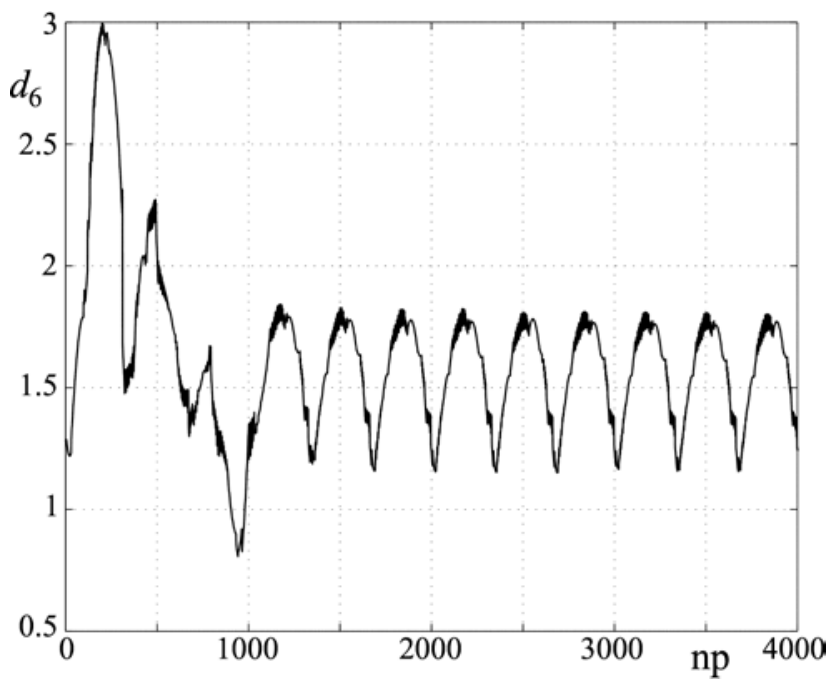

FIGURE 17. COMPUTED VALUES OF $d_{6}$ IN THE CIRCULAR TEST. 


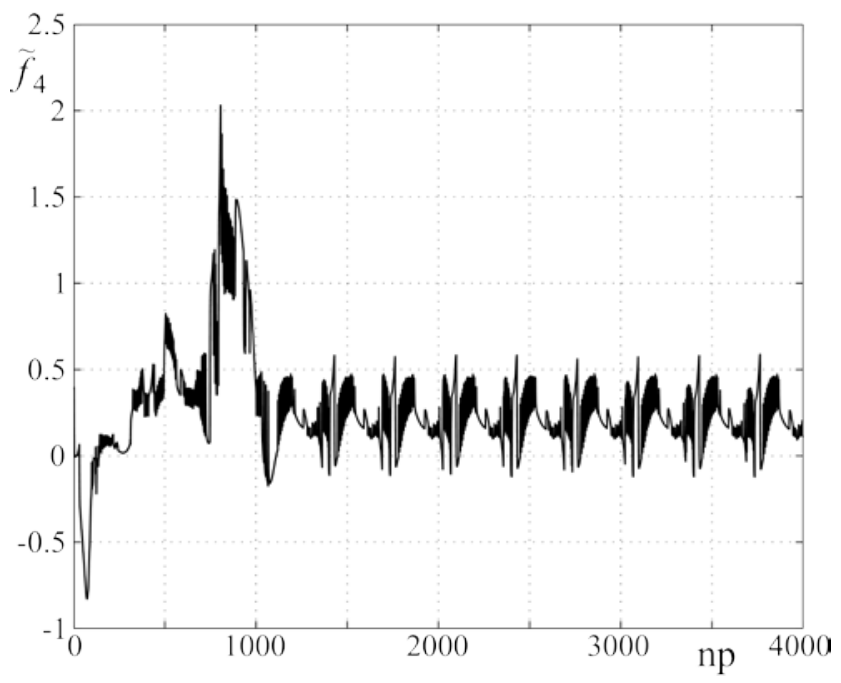

FIGURE 18. BEHAVIOR OF THE COEFFICIENT $\tilde{f}_{4}$ COMPUTED IN THE CIRCULAR TEST. 


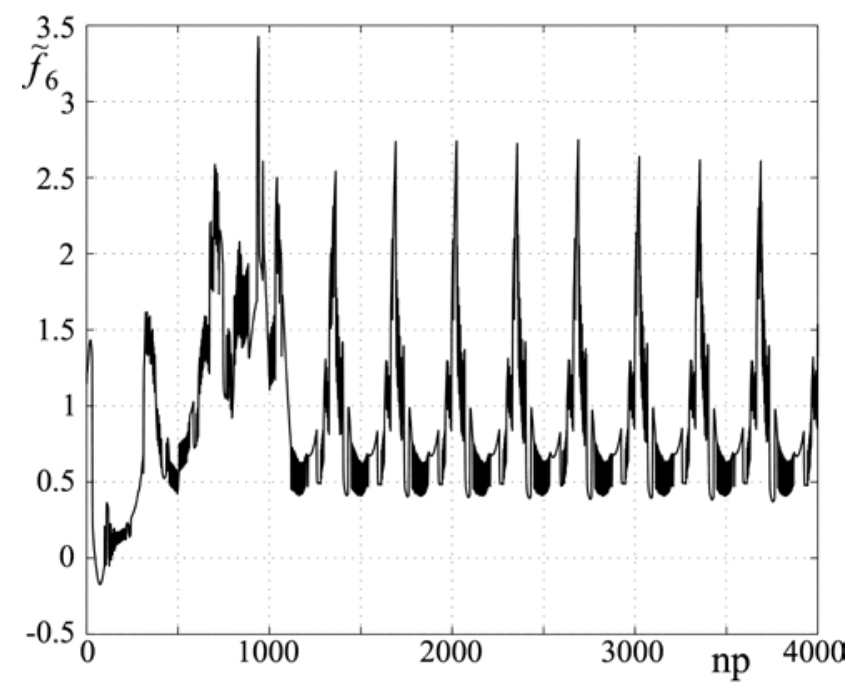

FIGURE 19. BEHAVIOR OF THE COEFFICIENT $\tilde{f}_{6}$ COMPUTED IN THE CIRCULAR TEST. 